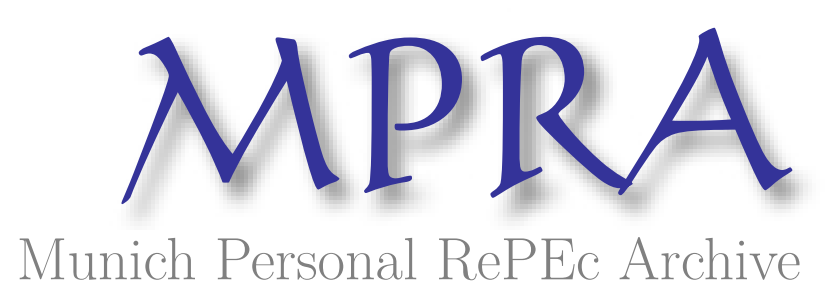

\title{
Mathematical Dynamics of Economic Growth as Effect of Internal Savings
}

Krouglov, Alexei

15 October 2006

Online at https://mpra.ub.uni-muenchen.de/1262/

MPRA Paper No. 1262, posted 28 Dec 2006 UTC 


\title{
Mathematical Dynamics of Economic Growth as Effect of Internal Savings
}

\author{
Alexei Krouglov \\ 796 Caboto Trail \\ Markham, Ontario L3R 4X1 Canada \\ alexkrouglov@concordidea.com
}




\title{
Mathematical Dynamics of Economic Growth as Effect of Internal Savings
}

\begin{abstract}
Paper introduces mathematical models describing long-time effects of real savings on economic growth. Models are built for single-product and multiple-product economy with market forces presented through the system of ordinary differential equations. Modeling results show a limited long-run economic growth for occasional and constant-rate systematic internal savings, a steady long-run economic growth if acceleration rate of internal savings lies within the proper limit for every industry, and a steady long-run economic decline if acceleration rate of internal savings exceeds the suitable limit for certain industry. Modeling outcome also suggests that a long-run economic growth requires direct investment of internal savings into appropriate investment vehicles with exclusion from savings-investment chain the interestrate-bearing bank accounts with clear danger of suffering a long-run economic decline in case of violation of the requirement.
\end{abstract}

Journal of Economic Literature Classification Numbers: E 32, O 41

Keywords: business fluctuations, economic growth, savings, investment 


\section{Introduction}

This paper is a continuation of my recent book [3] where I presented mathematical model describing economic forces acting on economic markets through the system of ordinary differential equations. Particularly in that book I built a dynamic model explaining the effect of economic forces on economic growth in market economy. The reason is that market participants withdraw some products from the markets as savings and use the withdrawn products in consecutive production as investments. That drives the products' prices on the markets up and at the same time it drives the amounts of products on the markets down. When the effect of increase in the products' prices exceeds the effect of decrease in the amounts of products one can observe the effect of economic growth whereas she can observe the effect of economic decline in the opposite situation. One important point is that products' savings are used as consecutive investments in order to increase in the products' quality. Thus increase in the products' prices during periods of economic growth is accompanied by the continuous increase in the products' quality.

In the current paper I look into various effects that savings and investments exert on the economy.

Firstly I investigate the concept by utilizing a simplified mathematical model of economy, which operates with single product. After concept becomes clear I extend the model on economy that operates with multiple products. As earlier in [2], [3] I describe the multi-product market economy with the help of Input-Output model of Wassily Leontief (see [4], [5]). Here I am using the Leontief model to describe how dynamic forces affecting supply and demand on the market for one particular product are influencing the markets for other products that are produced in multi-product economy. Technological factors in economy are assumed to be constant.

\section{Model of Single-Product Economy}

Here in this section I show how process of savings in a single-product economy affects the situation on the market of product, and creates an economic growth.

The concept of economic growth is presented through dual impact of changes in the product's prices and changes in the supply-demand equilibrium on the market of product. 
After supply-demand equilibrium on the market of product is reached, the economic growth is achieved through continuous improvement in the product's quality. To improve the product's quality one has to make an appropriate investment of product (remember, we are dealing with a single-product economy). That is done by applying the product's savings i.e. withdrawing an appropriate amount of product from the market. That process creates a temporal or permanent shortage of product on the market, which violates supply-demand equilibrium on the market of product, and drives the product's price up. In other words, when one pays enlarged price for an improved-quality product, she compensates from economic point of view for an increase in the product's price caused by withdrawal of appropriate amount of product from the market through the process of savings (and consecutive investment) in order to improve the quality of product.

On the other hand, withdrawing the product from the market in form of savings decreases available amount of product there. The reduced amount of product on the market is offset through an increased product' supply. Thus withdrawing the product from the market for investment and replenishing the product on the market by suppliers have opposite effects on the supply-demand equilibrium on market. As a result it could appear a surplus or shortage of product on the market at some point in time but market forces will try to bring market to new supply-demand equilibrium in the long term. Similarly withdrawing the product from the market in form of savings increases the product's price in the long term. These dual impact drive the monetary value of product (equal to the product's price multiplied by the product's quantity) on market in opposite directions - an enlarged price drives the monetary value up, if the product's quantity increases it drives the monetary value up, and if the product's quantity decreases it drives the monetary value down. When the monetary value increases in time one can talk about economic growth, and when the monetary value decreases in time she can speak about economic recession.

To turn to mathematical descriptions, when there are no disturbing economic forces, the market is in equilibrium position, i.e. the product's supply and demand are equal, and they are developing with a constant rate and the product's price is fixed.

When the balance between the product's supply and demand is broken, the product's market is experienced the economic forces, which act to bring the market to a new equilibrium position. 
These economic forces are described by the following ordinary differential equations regarding to the product's supply $V_{S}(t)$, demand $V_{D}(t)$, and price $P_{R}(t)$ (see [3]),

$$
\begin{aligned}
& \frac{d P_{R}(t)}{d t}=-\lambda_{P}\left(V_{S}(t)-V_{D}(t)\right) \\
& \frac{d^{2} V_{S}(t)}{d t^{2}}=\lambda_{S} \frac{d P_{R}(t)}{d t} \\
& \frac{d^{2} V_{D}(t)}{d t^{2}}=-\lambda_{D} \frac{d^{2} P_{R}(t)}{d t^{2}}
\end{aligned}
$$

In Equations (1.1-3) above the values $\lambda_{P}, \lambda_{S}, \lambda_{D} \geq 0$ are constants.

I assume that the market had been in equilibrium position until time $t=t_{0}$, the volumes of product's supply $V_{S}(t)$ and demand $V_{D}(t)$ on the market were equal, and they both were developing with constant rate $r_{D}^{0}$.

$$
\begin{aligned}
& V_{D}(t)=r_{D}^{0}\left(t-t_{0}\right)+V_{D}^{0} \\
& V_{S}(t)=V_{D}(t)
\end{aligned}
$$

where $V_{D}\left(t_{0}\right)=V_{D}^{0}$.

I present few scenarios describing the situation with product's saving.

\section{A One-Time Withdrawal of Product Savings from Market}

At some point in time $t=t_{0}$ the equilibrium situation was broken, and the amount of product equal to $\Delta_{R}>0$ was removed from the market,

$$
V_{S}(t)=\left\{\begin{array}{cc}
V_{D}(t), & t<t_{0} \\
V_{D}^{0}-\Delta_{R}, & t=t_{0}
\end{array}\right.
$$

where $0<\Delta_{R} \leq V_{D}^{0}$. 
That scenario increases at time $t=t_{0}$ the amount of savings $S_{R}(t)$ for the product,

$$
S_{R}(t)=\left\{\begin{array}{cc}
0, & t<t_{0} \\
\Delta_{R}, & t=t_{0}
\end{array}\right.
$$

where $S_{R}(t)=0$ for $t<t_{0}$.

From Equations $(1.1-3)$ the volume of product's surplus (or shortage) $\left[V_{S}(t)-V_{D}(t)\right]$ for $t>t_{0}$ is described by,

$$
\frac{d^{2}}{d t^{2}}\left(V_{S}(t)-V_{D}(t)\right)+\lambda_{P} \lambda_{D} \frac{d}{d t}\left(V_{S}(t)-V_{D}(t)\right)+\lambda_{P} \lambda_{S}\left(V_{S}(t)-V_{D}(t)\right)=0
$$

with the following initial conditions,

$$
\begin{aligned}
& V_{S}\left(t_{0}\right)-V_{D}\left(t_{0}\right)=-\Delta_{R}, \\
& \frac{d}{d t}\left(V_{S}\left(t_{0}\right)-V_{D}\left(t_{0}\right)\right)=0 .
\end{aligned}
$$

Initial conditions for the product's price $P_{R}(t)$ are $P_{R}\left(t_{0}\right)=P_{R}^{0}$ and $\frac{d P_{R}\left(t_{0}\right)}{d t}=\lambda_{P} \Delta_{R}$.

Similar to Equation (1.8) the product's price $P_{R}(t)$ is described by the following second-order ordinary differential equation for $t>t_{0}$,

$$
\frac{d^{2} P_{R}(t)}{d t^{2}}+\lambda_{P} \lambda_{D} \frac{d P_{R}(t)}{d t}+\lambda_{P} \lambda_{S} P_{R}(t)+C=0
$$

where $C=-\lambda_{P}\left(\lambda_{P} \lambda_{D} \Delta_{R}+\lambda_{S} P_{R}^{0}\right)$ is a constant.

If one uses the new variable $P_{1}(t)=P_{R}(t)-P_{R}^{0}-\frac{\lambda_{P} \lambda_{D}}{\lambda_{S}} \Delta_{R}$, Equation (1.9) becomes,

$$
\frac{d^{2} P_{1}(t)}{d t^{2}}+\lambda_{P} \lambda_{D} \frac{d P_{1}(t)}{d t}+\lambda_{P} \lambda_{S} P_{1}(t)=0
$$

Therefore the initial conditions for $P_{1}(t)$ are $P_{1}\left(t_{0}\right)=-\frac{\lambda_{P} \lambda_{D}}{\lambda_{S}} \Delta_{R}$ and $\frac{d P_{1}\left(t_{0}\right)}{d t}=\lambda_{P} \Delta_{R}$. 
Equations (1.8) and (1.10) have the same characteristic equations. And the roots of these characteristic equations are,

$$
k_{1,2}=-\frac{\lambda_{P} \lambda_{D}}{2} \pm \sqrt{\frac{\lambda_{P}^{2} \lambda_{D}^{2}}{4}-\lambda_{P} \lambda_{S}}
$$

(a) If $\frac{\lambda_{P}^{2} \lambda_{D}^{2}}{4}>\lambda_{P} \lambda_{S}$ the solution of Equation (1.8) is (see [6] - [8]),

$$
V_{S}(t)-V_{D}(t)=C_{1} \exp \left\{k_{1}\left(t-t_{0}\right)\right\}+C_{2} \exp \left\{k_{2}\left(t-t_{0}\right)\right\},
$$

where $C_{1}=\Delta_{R} \frac{k_{2}}{k_{1}-k_{2}}$ and $C_{2}=\Delta_{R} \frac{k_{1}}{k_{2}-k_{1}}$.

If inequality above holds, the solution of Equation (1.10) is

$$
P_{1}(t)=C_{3} \exp \left\{k_{1}\left(t-t_{0}\right)\right\}+C_{4} \exp \left\{k_{2}\left(t-t_{0}\right)\right\}
$$

where $C_{3}=\lambda_{P} \Delta_{R} \frac{1-\left(\lambda_{D} / \lambda_{S}\right) k_{2}}{k_{1}-k_{2}}$ and $C_{4}=\lambda_{P} \Delta_{R} \frac{1-\left(\lambda_{D} / \lambda_{S}\right) k_{1}}{k_{2}-k_{1}}$ are constants.

Since $k_{1}<0$ and $k_{2}<0$ it takes place $V_{S}(t)-V_{D}(t) \rightarrow 0$ and $P_{1}(t) \rightarrow 0$ for $t \rightarrow+\infty$.

Then it follows from the change of variable,

$$
P_{R}(t)=P_{1}(t)+P_{R}^{0}+\frac{\lambda_{P} \lambda_{D}}{\lambda_{S}} \Delta_{R}
$$

and it takes place for $t \rightarrow+\infty$,

$$
P_{R}(t) \rightarrow P_{R}^{0}+\frac{\lambda_{P} \lambda_{D}}{\lambda_{S}} \Delta_{R}
$$

Since $V_{D}\left(t_{0}\right)=V_{D}^{0}$ and $\frac{d V_{D}\left(t_{0}\right)}{d t}=r_{D}^{0}$ it takes place from Equation (1.3),

$$
V_{D}(t)=-\lambda_{D} P_{1}(t)+\left(r_{D}^{0}+\lambda_{P} \lambda_{D} \Delta_{R}\right)\left(t-t_{0}\right)+V_{D}^{0}-\frac{\lambda_{P} \lambda_{D}^{2}}{\lambda_{S}} \Delta_{R}
$$

Since $V_{S}(t)-V_{D}(t) \rightarrow 0$ it takes place $V_{S}(t) \rightarrow V_{D}(t)$ for $t \rightarrow+\infty$.

I calculate now the effect of the product's savings by comparing two monetary values of product quantity taken at the limit $t \rightarrow+\infty$ i.e. when the market of product comes to a new equilibrium. The first 
monetary value is the product $v_{R}=P_{R}(t) \times V_{D}(t)$ of the product's price $P_{R}(t)$ and the product's demand $V_{D}(t)$ at the limit after savings. The second monetary value is the product $\widetilde{v}_{R}=\widetilde{P}_{R}(t) \times \widetilde{V}_{D}(t)$ of the product's price $\widetilde{P}_{R}(t)=P_{R}^{0}$ and the product's demand $\widetilde{V}_{D}(t)=r_{D}^{0}\left(t-t_{0}\right)+V_{D}^{0}$ at the limit if there was no withdrawal of product from the market.

$$
\begin{aligned}
& \text { Since } \lim _{t \rightarrow+\infty}\left(\frac{P_{R}(t)}{\widetilde{P}_{R}(t)}\right)=1+\frac{\lambda_{P} \lambda_{D} \Delta_{R}}{\lambda_{S} P_{R}^{0}} \text { and } \lim _{t \rightarrow+\infty}\left(\frac{V_{D}(t)}{\widetilde{V}_{D}(t)}\right)=1+\frac{\lambda_{P} \lambda_{D} \Delta_{R}}{r_{D}^{0}} \text {, it takes place, } \\
& \lim _{t \rightarrow+\infty}\left(\frac{v_{R}(t)}{\widetilde{v}_{R}(t)}\right)=\lim _{t \rightarrow+\infty}\left(\frac{P_{R}(t) \times V_{D}(t)}{\widetilde{P}_{R}(t) \times \widetilde{V}_{D}(t)}\right)=1+\frac{\lambda_{P} \lambda_{D} \Delta_{R}}{\lambda_{S} P_{R}^{0} r_{D}^{0}}\left(\lambda_{S} P_{R}^{0}+r_{D}^{0}+\lambda_{P} \lambda_{D} \Delta_{R}\right)
\end{aligned}
$$

(b) If $\frac{\lambda_{P}^{2} \lambda_{D}^{2}}{4}=\lambda_{P} \lambda_{S}$ the solution of Equation (1.8) is (see [6]-[8]),

$$
V_{S}(t)-V_{D}(t)=\left(C_{1}+C_{2}\left(t-t_{0}\right)\right) \exp \left\{-\frac{\lambda_{P} \lambda_{D}}{2}\left(t-t_{0}\right)\right\}
$$

where $C_{1}=-\Delta_{R}$ and $C_{2}=-\frac{\lambda_{P} \lambda_{D}}{2} \Delta_{R}$

If equality above holds, the solution of Equation (1.10) is

$$
P_{1}(t)=\left(C_{3}+C_{4}\left(t-t_{0}\right)\right) \exp \left\{-\frac{\lambda_{P} \lambda_{D}}{2}\left(t-t_{0}\right)\right\},
$$

where $C_{3}=-\frac{\lambda_{P} \lambda_{D}}{\lambda_{S}} \Delta_{R}$ and $C_{4}=-\lambda_{P} \Delta_{R}$ are constants.

Since $\lambda_{P} \lambda_{D}>0$ it takes place $V_{S}(t)-V_{D}(t) \rightarrow 0$ and $P_{1}(t) \rightarrow 0$ for $t \rightarrow+\infty$.

Therefore it takes place for $t \rightarrow+\infty$ as before,

$$
\begin{gathered}
P_{R}(t) \rightarrow P_{R}^{0}+\frac{\lambda_{P} \lambda_{D}}{\lambda_{S}} \Delta_{R} \\
V_{D}(t)=-\lambda_{D} P_{1}(t)+\left(r_{D}^{0}+\lambda_{P} \lambda_{D} \Delta_{R}\right)\left(t-t_{0}\right)+V_{D}^{0}-\frac{\lambda_{P} \lambda_{D}^{2}}{\lambda_{S}} \Delta_{R}
\end{gathered}
$$

and $V_{S}(t) \rightarrow V_{D}(t)$ for $t \rightarrow+\infty$. 


$$
\begin{aligned}
& \text { Since } \lim _{t \rightarrow+\infty}\left(\frac{P_{R}(t)}{\widetilde{P}_{R}(t)}\right)=1+\frac{\lambda_{P} \lambda_{D} \Delta_{R}}{\lambda_{S} P_{R}^{0}} \text { and } \lim _{t \rightarrow+\infty}\left(\frac{V_{D}(t)}{\widetilde{V}_{D}(t)}\right)=1+\frac{\lambda_{P} \lambda_{D} \Delta_{R}}{r_{D}^{0}} \text {, it takes place, } \\
& \lim _{t \rightarrow+\infty}\left(\frac{v_{R}(t)}{\widetilde{v}_{R}(t)}\right)=\lim _{t \rightarrow+\infty}\left(\frac{P_{R}(t) \times V_{D}(t)}{\widetilde{P}_{R}(t) \times \widetilde{V}_{D}(t)}\right)=1+\frac{\lambda_{P} \lambda_{D} \Delta_{R}}{\lambda_{S} P_{R}^{0} r_{D}^{0}}\left(\lambda_{S} P_{R}^{0}+r_{D}^{0}+\lambda_{P} \lambda_{D} \Delta_{R}\right)
\end{aligned}
$$

(c) If $\frac{\lambda_{P}^{2} \lambda_{D}^{2}}{4}<\lambda_{P} \lambda_{S}$ the solution of Equation (1.8) is (see [6] - [8]),

$V_{S}(t)-V_{D}(t)=\exp \left\{-\frac{\lambda_{P} \lambda_{D}}{2}\left(t-t_{0}\right)\right\}\left(C_{1} \cos \left(\sqrt{\lambda_{P} \lambda_{S}-\frac{\lambda_{P}^{2} \lambda_{D}^{2}}{4}}\left(t-t_{0}\right)\right)+C_{2} \sin \left(\sqrt{\lambda_{P} \lambda_{S}-\frac{\lambda_{P}^{2} \lambda_{D}^{2}}{4}}\left(t-t_{0}\right)\right)\right)$

where $C_{1}=-\Delta_{R}$ and $C_{2}=\frac{-\lambda_{P} \lambda_{D} \Delta_{R}}{2 \sqrt{\lambda_{P} \lambda_{S}-\frac{\lambda_{P}^{2} \lambda_{D}^{2}}{4}}}$

If inequality above holds, the solution of Equation (1.10) is

$$
P_{1}(t)=\exp \left\{-\frac{\lambda_{P} \lambda_{D}}{2}\left(t-t_{0}\right)\right\}\left(C_{3} \cos \left(\sqrt{\lambda_{P} \lambda_{S}-\frac{\lambda_{P}^{2} \lambda_{D}^{2}}{4}}\left(t-t_{0}\right)\right)+C_{4} \sin \left(\sqrt{\lambda_{P} \lambda_{S}-\frac{\lambda_{P}^{2} \lambda_{D}^{2}}{4}}\left(t-t_{0}\right)\right)\right)
$$

where $C_{3}=-\frac{\lambda_{P} \lambda_{D}}{\lambda_{S}} \Delta_{R}$ and $C_{4}=\frac{\lambda_{P} \Delta_{R}}{\sqrt{\lambda_{P} \lambda_{S}-\frac{\lambda_{P}^{2} \lambda_{D}^{2}}{4}}}\left(1-\frac{\lambda_{P} \lambda_{D}^{2}}{2 \lambda_{S}}\right)$ are constants.

Since $\lambda_{P} \lambda_{D}>0$ it takes place $V_{S}(t)-V_{D}(t) \rightarrow 0$ and $P_{1}(t) \rightarrow 0$ for $t \rightarrow+\infty$.

Therefore it takes place for $t \rightarrow+\infty$ as before,

$$
\begin{gathered}
P_{R}(t) \rightarrow P_{R}^{0}+\frac{\lambda_{P} \lambda_{D}}{\lambda_{S}} \Delta_{R} \\
V_{D}(t)=-\lambda_{D} P_{1}(t)+\left(r_{D}^{0}+\lambda_{P} \lambda_{D} \Delta_{R}\right)\left(t-t_{0}\right)+V_{D}^{0}-\frac{\lambda_{P} \lambda_{D}^{2}}{\lambda_{S}} \Delta_{R}
\end{gathered}
$$

and $V_{S}(t) \rightarrow V_{D}(t)$ for $t \rightarrow+\infty$. 


$$
\begin{aligned}
& \text { Since } \lim _{t \rightarrow+\infty}\left(\frac{P_{R}(t)}{\widetilde{P}_{R}(t)}\right)=1+\frac{\lambda_{P} \lambda_{D} \Delta_{R}}{\lambda_{S} P_{R}^{0}} \text { and } \lim _{t \rightarrow+\infty}\left(\frac{V_{D}(t)}{\widetilde{V}_{D}(t)}\right)=1+\frac{\lambda_{P} \lambda_{D} \Delta_{R}}{r_{D}^{0}} \text {, it takes place, } \\
& \lim _{t \rightarrow+\infty}\left(\frac{v_{R}(t)}{\widetilde{v}_{R}(t)}\right)=\lim _{t \rightarrow+\infty}\left(\frac{P_{R}(t) \times V_{D}(t)}{\widetilde{P}_{R}(t) \times \widetilde{V}_{D}(t)}\right)=1+\frac{\lambda_{P} \lambda_{D} \Delta_{R}}{\lambda_{S} P_{R}^{0} r_{D}^{0}}\left(\lambda_{S} P_{R}^{0}+r_{D}^{0}+\lambda_{P} \lambda_{D} \Delta_{R}\right)
\end{aligned}
$$

Thus all cases to deduce the solutions of differential equations from the roots of related characteristic equation are covered.

Therefore at the limit for $t \rightarrow+\infty$ the withdrawal of product from the market causes both an increase of the product's price and an increase of product's demand. These actions raise the monetary value $v_{R}(t)$ of product on the market in the long run,

$$
\begin{aligned}
& \lim _{t \rightarrow+\infty}\left(\frac{P_{R}(t)}{\widetilde{P}_{R}(t)}\right)=1+\frac{\lambda_{P} \lambda_{D} \Delta_{R}}{\lambda_{S} P_{R}^{0}}>1 \\
& \lim _{t \rightarrow+\infty}\left(\frac{V_{D}(t)}{\widetilde{V}_{D}(t)}\right)=1+\frac{\lambda_{P} \lambda_{D} \Delta_{R}}{r_{D}^{0}}>1 \\
& \lim _{t \rightarrow+\infty}\left(\frac{v_{R}(t)}{\widetilde{v}_{R}(t)}\right)=1+\frac{\lambda_{P} \lambda_{D} \Delta_{R}}{\lambda_{S} P_{R}^{0} r_{D}^{0}}\left(\lambda_{S} P_{R}^{0}+r_{D}^{0}+\lambda_{P} \lambda_{D} \Delta_{R}\right)>1
\end{aligned}
$$

That concludes the first scenario.

\section{B Constant-Rate Continuous Withdrawal of Product Savings from Market}

According to this scenario I assume that amount of product's savings $S_{R}(t)$ increases since time $t=t_{0}$ according to following formula,

$$
S_{R}(t)=\left\{\begin{array}{cc}
0, & t<t_{0} \\
\delta_{R}\left(t-t_{0}\right), & t \geq t_{0}
\end{array}\right.
$$

where $S_{R}(t)=0$ for $t<t_{0}$ and $\delta_{R}>0$. 
Therefore taken into account the product's withdrawal from the market in the form of product's savings $S_{R}(t)$ described by Equation (1.28) the volume of product's surplus (or shortage) on the market $\left[V_{S}(t)-V_{D}(t)\right]$ in Equations $(1.1-3)$ has to be replaced by the volume of product's surplus (or shortage) on the market expressed as $D_{R} \equiv\left(V_{S}(t)-V_{D}(t)-S_{R}(t)\right)$; that produces for $t>t_{0}$,

$$
\frac{d^{2} D_{R}(t)}{d t^{2}}+\lambda_{P} \lambda_{D} \frac{d D_{R}(t)}{d t}+\lambda_{P} \lambda_{S} D_{R}(t)=0
$$

with the following initial conditions,

$$
\begin{aligned}
& D_{R}\left(t_{0}\right)=0, \\
& \frac{d D_{R}\left(t_{0}\right)}{d t}=-\delta_{R} .
\end{aligned}
$$

Initial conditions for the product's price $P_{R}(t)$ are $P_{R}\left(t_{0}\right)=P_{R}^{0}$ and $\frac{d P_{R}\left(t_{0}\right)}{d t}=0$.

Similar to Equation (1.29) the product's price $P_{R}(t)$ is described by the following second-order ordinary differential equation for $t>t_{0}$,

$$
\frac{d^{2} P_{R}(t)}{d t^{2}}+\lambda_{P} \lambda_{D} \frac{d P_{R}(t)}{d t}+\lambda_{P} \lambda_{S} P_{R}(t)+C=0
$$

where $C=-\lambda_{P}\left(\lambda_{S} P_{R}^{0}+\delta_{R}\right)$ is a constant.

If one uses the new variable $P_{1}(t)=P_{R}(t)-P_{R}^{0}-\frac{1}{\lambda_{S}} \delta_{R}$, Equation (1.30) becomes,

$$
\frac{d^{2} P_{1}(t)}{d t^{2}}+\lambda_{P} \lambda_{D} \frac{d P_{1}(t)}{d t}+\lambda_{P} \lambda_{S} P_{1}(t)=0
$$

Therefore the initial conditions for $P_{1}(t)$ are $P_{1}\left(t_{0}\right)=-\frac{1}{\lambda_{S}} \delta_{R}$ and $\frac{d P_{1}\left(t_{0}\right)}{d t}=0$.

Equations (1.29) and (1.31) have the same characteristic equations. And the roots of these characteristic equations are,

$$
k_{1,2}=-\frac{\lambda_{P} \lambda_{D}}{2} \pm \sqrt{\frac{\lambda_{P}^{2} \lambda_{D}^{2}}{4}-\lambda_{P} \lambda_{S}}
$$


(a) If $\frac{\lambda_{P}^{2} \lambda_{D}^{2}}{4}>\lambda_{P} \lambda_{S}$ the solution of Equation (1.29) is,

$$
D_{R}(t)=C_{1} \exp \left\{k_{1}\left(t-t_{0}\right)\right\}+C_{2} \exp \left\{k_{2}\left(t-t_{0}\right)\right\}
$$

where $C_{1}=\frac{\delta_{R}}{k_{2}-k_{1}}$ and $C_{2}=\frac{\delta_{R}}{k_{1}-k_{2}}$.

If inequality above holds, the solution of Equation (1.31) is

$$
P_{1}(t)=C_{3} \exp \left\{k_{1}\left(t-t_{0}\right)\right\}+C_{4} \exp \left\{k_{2}\left(t-t_{0}\right)\right\}
$$

where $C_{3}=\frac{\delta_{R}}{\lambda_{S}} \cdot \frac{k_{2}}{k_{1}-k_{2}}$ and $C_{4}=\frac{\delta_{R}}{\lambda_{S}} \cdot \frac{k_{1}}{k_{2}-k_{1}}$ are constants.

Since $k_{1}<0$ and $k_{2}<0$ it takes place $D_{R}(t) \rightarrow 0$ and $P_{1}(t) \rightarrow 0$ for $t \rightarrow+\infty$.

Then it follows from the change of variable,

$$
P_{R}(t)=P_{1}(t)+P_{R}^{0}+\frac{1}{\lambda_{S}} \delta_{R}
$$

and it takes place for $t \rightarrow+\infty$,

$$
P_{R}(t) \rightarrow P_{R}^{0}+\frac{1}{\lambda_{S}} \delta_{R}
$$

Since $V_{S}\left(t_{0}\right)=V_{D}^{0}$ and $\frac{d V_{S}\left(t_{0}\right)}{d t}=r_{D}^{0}$ it takes place from Equations $(1.1-3)$,

$$
V_{S}(t)=\lambda_{S} \frac{C_{3}}{k_{1}} \exp \left\{k_{1}\left(t-t_{0}\right)\right\}+\lambda_{S} \frac{C_{4}}{k_{2}} \exp \left\{k_{2}\left(t-t_{0}\right)\right\}+\left(r_{D}^{0}+\delta_{R}\right)\left(t-t_{0}\right)+V_{D}^{0}-\frac{\lambda_{D}}{\lambda_{S}} \delta_{R}
$$

and it follows from Equations (1.28), (1.33), (1.37) for $t \rightarrow+\infty$,

$$
\begin{aligned}
& V_{S}(t) \rightarrow\left(r_{D}^{0}+\delta_{R}\right)\left(t-t_{0}\right)+V_{D}^{0}-\frac{\lambda_{D}}{\lambda_{S}} \delta_{R} \\
& V_{D}(t) \rightarrow r_{D}^{0}\left(t-t_{0}\right)+V_{D}^{0}-\frac{\lambda_{D}}{\lambda_{S}} \delta_{R} \\
& S_{R}(t)=\delta_{R}\left(t-t_{0}\right)
\end{aligned}
$$




$$
\begin{aligned}
& \text { Since } \lim _{t \rightarrow+\infty}\left(\frac{P_{R}(t)}{\widetilde{P}_{R}(t)}\right)=1+\frac{\delta_{R}}{\lambda_{S} P_{R}^{0}} \text { and } \lim _{t \rightarrow+\infty}\left(\frac{V_{D}(t)}{\widetilde{V}_{D}(t)}\right)=1, \text { it takes place, } \\
& \lim _{t \rightarrow+\infty}\left(\frac{v_{R}(t)}{\widetilde{v}_{R}(t)}\right)=\lim _{t \rightarrow+\infty}\left(\frac{P_{R}(t) \times V_{D}(t)}{\widetilde{P}_{R}(t) \times \widetilde{V}_{D}(t)}\right)=1+\frac{\delta_{R}}{\lambda_{S} P_{R}^{0}}
\end{aligned}
$$

(b) If $\frac{\lambda_{P}^{2} \lambda_{D}^{2}}{4}=\lambda_{P} \lambda_{S}$ the solution of Equation (1.29) is,

$$
D_{R}(t)=\left(C_{1}+C_{2}\left(t-t_{0}\right)\right) \exp \left\{-\frac{\lambda_{P} \lambda_{D}}{2}\left(t-t_{0}\right)\right\}
$$

where $C_{1}=0$ and $C_{2}=-\delta_{R}$.

If equality above holds, the solution of Equation (1.31) is

$$
P_{1}(t)=\left(C_{3}+C_{4}\left(t-t_{0}\right)\right) \exp \left\{-\frac{\lambda_{P} \lambda_{D}}{2}\left(t-t_{0}\right)\right\}
$$

where $C_{3}=-\frac{1}{\lambda_{S}} \delta_{R}$ and $C_{4}=-\frac{2}{\lambda_{D}} \delta_{R}$ are constants.

Since $\lambda_{P} \lambda_{D}>0$ it takes place $D_{R}(t) \rightarrow 0$ and $P_{1}(t) \rightarrow 0$ for $t \rightarrow+\infty$.

Then it follows from the change of variable,

$$
P_{R}(t)=P_{1}(t)+P_{R}^{0}+\frac{1}{\lambda_{S}} \delta_{R}
$$

and it takes place for $t \rightarrow+\infty$,

$$
P_{R}(t) \rightarrow P_{R}^{0}+\frac{1}{\lambda_{S}} \delta_{R}
$$

Since $V_{S}\left(t_{0}\right)=V_{D}^{0}$ and $\frac{d V_{S}\left(t_{0}\right)}{d t}=r_{D}^{0}$ it takes place from Equations $(1.1-3)$,

$V_{S}(t)=\left(-\frac{\lambda_{D}}{2} C_{3}-\frac{1}{\lambda_{P}} C_{4}\left(\frac{\lambda_{P} \lambda_{D}}{2}\left(t-t_{0}\right)+1\right)\right) \exp \left\{-\frac{\lambda_{P} \lambda_{D}}{2}\left(t-t_{0}\right)\right\}+\left(r_{D}^{0}+\delta_{R}\right)\left(t-t_{0}\right)+V_{D}^{0}-\frac{\lambda_{D}}{\lambda_{S}} \delta_{R}$

and it follows from Equations (1.28), (1.42), (1.46) for $t \rightarrow+\infty$, 


$$
\begin{aligned}
& V_{S}(t) \rightarrow\left(r_{D}^{0}+\delta_{R}\right)\left(t-t_{0}\right)+V_{D}^{0}-\frac{\lambda_{D}}{\lambda_{S}} \delta_{R} \\
& V_{D}(t) \rightarrow r_{D}^{0}\left(t-t_{0}\right)+V_{D}^{0}-\frac{\lambda_{D}}{\lambda_{S}} \delta_{R} \\
& S_{R}(t)=\delta_{R}\left(t-t_{0}\right)
\end{aligned}
$$

Since $\lim _{t \rightarrow+\infty}\left(\frac{P_{R}(t)}{\widetilde{P}_{R}(t)}\right)=1+\frac{\delta_{R}}{\lambda_{S} P_{R}^{0}}$ and $\lim _{t \rightarrow+\infty}\left(\frac{V_{D}(t)}{\widetilde{V}_{D}(t)}\right)=1$, it takes place,

$$
\lim _{t \rightarrow+\infty}\left(\frac{v_{R}(t)}{\widetilde{v}_{R}(t)}\right)=\lim _{t \rightarrow+\infty}\left(\frac{P_{R}(t) \times V_{D}(t)}{\widetilde{P}_{R}(t) \times \widetilde{V}_{D}(t)}\right)=1+\frac{\delta_{R}}{\lambda_{S} P_{R}^{0}}
$$

(c) If $\frac{\lambda_{P}^{2} \lambda_{D}^{2}}{4}<\lambda_{P} \lambda_{S}$ the solution of Equation (1.29) is,

$D_{R}(t)=\exp \left\{-\frac{\lambda_{P} \lambda_{D}}{2}\left(t-t_{0}\right)\right\}\left(C_{1} \cos \left(\sqrt{\lambda_{P} \lambda_{S}-\frac{\lambda_{P}^{2} \lambda_{D}^{2}}{4}}\left(t-t_{0}\right)\right)+C_{2} \sin \left(\sqrt{\lambda_{P} \lambda_{S}-\frac{\lambda_{P}^{2} \lambda_{D}^{2}}{4}}\left(t-t_{0}\right)\right)\right)$

where $C_{1}=0$ and $C_{2}=\frac{-\delta_{R}}{\sqrt{\lambda_{P} \lambda_{S}-\frac{\lambda_{P}^{2} \lambda_{D}^{2}}{4}}}$

If inequality above holds, the solution of Equation (1.31) is

$$
P_{1}(t)=\exp \left\{-\frac{\lambda_{P} \lambda_{D}}{2}\left(t-t_{0}\right)\right\}\left(C_{3} \cos \left(\sqrt{\lambda_{P} \lambda_{S}-\frac{\lambda_{P}^{2} \lambda_{D}^{2}}{4}}\left(t-t_{0}\right)\right)+C_{4} \sin \left(\sqrt{\lambda_{P} \lambda_{S}-\frac{\lambda_{P}^{2} \lambda_{D}^{2}}{4}}\left(t-t_{0}\right)\right)\right)
$$

where $C_{3}=-\frac{1}{\lambda_{S}} \delta_{R}$ and $C_{4}=-\frac{\lambda_{P} \lambda_{D}}{\lambda_{S}} \cdot \frac{\delta_{R}}{2 \sqrt{\lambda_{P} \lambda_{S}-\frac{\lambda_{P}^{2} \lambda_{D}^{2}}{4}}}$ are constants.

Since $\lambda_{P} \lambda_{D}>0$ it takes place $D_{R}(t) \rightarrow 0$ and $P_{1}(t) \rightarrow 0$ for $t \rightarrow+\infty$.

Then it follows from the change of variable,

$$
P_{R}(t)=P_{1}(t)+P_{R}^{0}+\frac{1}{\lambda_{S}} \delta_{R}
$$


and it takes place for $t \rightarrow+\infty$,

$$
P_{R}(t) \rightarrow P_{R}^{0}+\frac{1}{\lambda_{S}} \delta_{R}
$$

Since $V_{S}\left(t_{0}\right)=V_{D}^{0}$ and $\frac{d V_{S}\left(t_{0}\right)}{d t}=r_{D}^{0}$ it takes place from Equations $(1.1-3)$,

$V_{S}(t)=\frac{1}{\lambda_{P}} \cdot \exp \left\{-\frac{\lambda_{P} \lambda_{D}}{2}\left(t-t_{0}\right)\right\} \times\left(\begin{array}{l}-\left(C_{3} \cdot \frac{\lambda_{P} \lambda_{D}}{2}+C_{4} \cdot \sqrt{\lambda_{P} \lambda_{S}-\frac{\lambda_{P}^{2} \lambda_{D}^{2}}{4}}\right) \cdot \cos \left(\sqrt{\lambda_{P} \lambda_{S}-\frac{\lambda_{P}^{2} \lambda_{D}^{2}}{4}}\left(t-t_{0}\right)\right) \\ +\left(C_{3} \cdot \sqrt{\lambda_{P} \lambda_{S}-\frac{\lambda_{P}^{2} \lambda_{D}^{2}}{4}}-C_{4} \cdot \frac{\lambda_{P} \lambda_{D}}{2}\right) \cdot \sin \left(\sqrt{\lambda_{P} \lambda_{S}-\frac{\lambda_{P}^{2} \lambda_{D}^{2}}{4}}\left(t-t_{0}\right)\right)\end{array}\right)$

$+\left(r_{D}^{0}+\delta_{R}\right)\left(t-t_{0}\right)+V_{D}^{0}-\frac{\lambda_{D}}{\lambda_{S}} \delta_{R}$

and it follows from Equations (1.28), (1.51), (1.55) for $t \rightarrow+\infty$,

$$
\begin{aligned}
& V_{S}(t) \rightarrow\left(r_{D}^{0}+\delta_{R}\right)\left(t-t_{0}\right)+V_{D}^{0}-\frac{\lambda_{D}}{\lambda_{S}} \delta_{R} \\
& V_{D}(t) \rightarrow r_{D}^{0}\left(t-t_{0}\right)+V_{D}^{0}-\frac{\lambda_{D}}{\lambda_{S}} \delta_{R} \\
& S_{R}(t)=\delta_{R}\left(t-t_{0}\right) \\
& \text { Since } \lim _{t \rightarrow+\infty}\left(\frac{P_{R}(t)}{\widetilde{P}_{R}(t)}\right)=1+\frac{\delta_{R}}{\lambda_{S} P_{R}^{0}} \text { and } \lim _{t \rightarrow+\infty}\left(\frac{V_{D}(t)}{\widetilde{V}_{D}(t)}\right)=1 \text {, it takes place, } \\
& \lim _{t \rightarrow+\infty}\left(\frac{v_{R}(t)}{\widetilde{v}_{R}(t)}\right)=\lim _{t \rightarrow+\infty}\left(\frac{P_{R}(t) \times V_{D}(t)}{\widetilde{P}_{R}(t) \times \widetilde{V}_{D}(t)}\right)=1+\frac{\delta_{R}}{\lambda_{S} P_{R}^{0}}
\end{aligned}
$$

Thus all cases to deduce the solutions of differential equations from the roots of related characteristic equation are covered.

Therefore at the limit for $t \rightarrow+\infty$ the withdrawal of product from the market causes an increase of the product's price and practically doesn't change the product's demand. These actions raise the monetary value $v_{R}(t)$ of product on the market in the long run, 


$$
\begin{aligned}
& \lim _{t \rightarrow+\infty}\left(\frac{P_{R}(t)}{\widetilde{P}_{R}(t)}\right)=1+\frac{\delta_{R}}{\lambda_{S} P_{R}^{0}}>1 \\
& \lim _{t \rightarrow+\infty}\left(\frac{V_{D}(t)}{\widetilde{V}_{D}(t)}\right)=1 \\
& \lim _{t \rightarrow+\infty}\left(\frac{v_{R}(t)}{\widetilde{v}_{R}(t)}\right)=1+\frac{\delta_{R}}{\lambda_{S} P_{R}^{0}}>1
\end{aligned}
$$

That concludes the second scenario.

\section{Constant-Accelerated Continuous Withdrawal of Product Savings from Market}

According to this scenario I assume that amount of product's savings $S_{R}(t)$ increases since time $t=t_{0}$ according to following formula,

$$
S_{R}(t)=\left\{\begin{array}{cc}
0, & t<t_{0} \\
\delta_{R}\left(t-t_{0}\right)+\frac{\varepsilon_{R}}{2}\left(t-t_{0}\right)^{2}, & t \geq t_{0}
\end{array}\right.
$$

where $S_{R}(t)=0$ for $t<t_{0}, \delta_{R}>0$, and $\varepsilon_{R}>0$.

Therefore taken into account the product's withdrawal from the market in the form of product's savings $S_{R}(t)$ described by Equation (1.60) the volume of product's surplus (or shortage) on the market $\left[V_{S}(t)-V_{D}(t)\right]$ in Equations $(1.1-3)$ has to be replaced by the volume of product's surplus (or shortage) on the market expressed as $D_{R} \equiv\left(V_{S}(t)-V_{D}(t)-S_{R}(t)\right)$; that produces for $t>t_{0}$,

$$
\frac{d^{2} D_{R}(t)}{d t^{2}}+\lambda_{P} \lambda_{D} \frac{d D_{R}(t)}{d t}+\lambda_{P} \lambda_{S} D_{R}(t)+\varepsilon_{R}=0
$$

with the following initial conditions,

$$
D_{R}\left(t_{0}\right)=0 \text {, }
$$


$\frac{d D_{R}\left(t_{0}\right)}{d t}=-\delta_{R}$

If one uses the new variable $D_{1}(t)=D_{R}(t)+\frac{1}{\lambda_{P} \lambda_{S}} \varepsilon_{R}$, then Equation (1.61) becomes,

$$
\frac{d^{2} D_{1}(t)}{d t^{2}}+\lambda_{P} \lambda_{D} \frac{d D_{1}(t)}{d t}+\lambda_{P} \lambda_{S} D_{1}(t)=0
$$

with the following initial conditions,

$$
\begin{aligned}
& D_{1}\left(t_{0}\right)=\frac{1}{\lambda_{P} \lambda_{S}} \varepsilon_{R}, \\
& \frac{d D_{1}\left(t_{0}\right)}{d t}=-\delta_{R} .
\end{aligned}
$$

Initial conditions for the product's price $P_{R}(t)$ are $P_{R}\left(t_{0}\right)=P_{R}^{0}$ and $\frac{d P_{R}\left(t_{0}\right)}{d t}=0$.

Similar to Equation (1.61) the product's price $P_{R}(t)$ is described by the following second-order ordinary differential equation for $t>t_{0}$,

$$
\frac{d^{2} P_{R}(t)}{d t^{2}}+\lambda_{P} \lambda_{D} \frac{d P_{R}(t)}{d t}+\lambda_{P} \lambda_{S} P_{R}(t)-\lambda_{P}\left(\varepsilon_{R}\left(t-t_{0}\right)+\delta_{R}+\lambda_{S} P_{R}^{0}\right)=0
$$

If one uses the new variable $P_{1}(t)=P_{R}(t)-P_{R}^{0}-\frac{1}{\lambda_{S}} \delta_{R}-\frac{1}{\lambda_{S}} \varepsilon_{R}\left(t-t_{0}\right)+\frac{\lambda_{D}}{\lambda_{S}^{2}} \varepsilon_{R}$, then Equation (1.63) becomes,

$$
\frac{d^{2} P_{1}(t)}{d t^{2}}+\lambda_{P} \lambda_{D} \frac{d P_{1}(t)}{d t}+\lambda_{P} \lambda_{S} P_{1}(t)=0
$$

Therefore initial conditions for $P_{1}(t)$ are $P_{1}\left(t_{0}\right)=-\frac{1}{\lambda_{S}} \delta_{R}+\frac{\lambda_{D}}{\lambda_{S}^{2}} \varepsilon_{R}$ and $\frac{d P_{1}\left(t_{0}\right)}{d t}=-\frac{1}{\lambda_{S}} \varepsilon_{R}$.

Equations (1.62) and (1.64) have the same characteristic equations. And the roots of these characteristic equations are,

$$
k_{1,2}=-\frac{\lambda_{P} \lambda_{D}}{2} \pm \sqrt{\frac{\lambda_{P}^{2} \lambda_{D}^{2}}{4}-\lambda_{P} \lambda_{S}}
$$


(a) If $\frac{\lambda_{P}^{2} \lambda_{D}^{2}}{4}>\lambda_{P} \lambda_{S}$ the solution of Equation (1.62) is,

$$
D_{1}(t)=C_{1} \exp \left\{k_{1}\left(t-t_{0}\right)\right\}+C_{2} \exp \left\{k_{2}\left(t-t_{0}\right)\right\},
$$

where $C_{1}=\frac{\delta_{R}+\frac{\varepsilon_{R}}{\lambda_{P} \lambda_{S}} k_{2}}{k_{2}-k_{1}}$ and $C_{2}=\frac{\delta_{R}+\frac{\varepsilon_{R}}{\lambda_{P} \lambda_{S}} k_{1}}{k_{1}-k_{2}}$

If inequality above holds, the solution of Equation (1.64) is

$$
P_{1}(t)=C_{3} \exp \left\{k_{1}\left(t-t_{0}\right)\right\}+C_{4} \exp \left\{k_{2}\left(t-t_{0}\right)\right\}
$$

where $\quad C_{3}=\frac{-\frac{1}{\lambda_{S}} \varepsilon_{R}+\left(\frac{1}{\lambda_{S}} \delta_{R}-\frac{\lambda_{D}}{\lambda_{S}^{2}} \varepsilon_{R}\right) k_{2}}{k_{1}-k_{2}} \quad$ and $\quad C_{4}=\frac{-\frac{1}{\lambda_{S}} \varepsilon_{R}+\left(\frac{1}{\lambda_{S}} \delta_{R}-\frac{\lambda_{D}}{\lambda_{S}^{2}} \varepsilon_{R}\right) k_{1}}{k_{2}-k_{1}} \quad$ are constants.

Since $k_{1}<0$ and $k_{2}<0$ it takes place $D_{1}(t) \rightarrow 0$ and $P_{1}(t) \rightarrow 0$ for $t \rightarrow+\infty$.

Then it follows from the change of variable,

$$
\begin{aligned}
& D_{R}(t)=D_{1}(t)-\frac{1}{\lambda_{P} \lambda_{S}} \varepsilon_{R} \\
& P_{R}(t)=P_{1}(t)+P_{R}^{0}+\frac{1}{\lambda_{S}} \delta_{R}+\frac{1}{\lambda_{S}} \varepsilon_{R}\left(t-t_{0}\right)-\frac{\lambda_{D}}{\lambda_{S}^{2}} \varepsilon_{R}
\end{aligned}
$$

and it takes place for $t \rightarrow+\infty$,

$$
\begin{aligned}
& D_{R}(t) \rightarrow-\frac{1}{\lambda_{P} \lambda_{S}} \varepsilon_{R} \\
& P_{R}(t) \rightarrow \frac{1}{\lambda_{S}} \varepsilon_{R}\left(t-t_{0}\right)+P_{R}^{0}+\frac{1}{\lambda_{S}} \delta_{R}-\frac{\lambda_{D}}{\lambda_{S}^{2}} \varepsilon_{R}
\end{aligned}
$$

Since $V_{S}\left(t_{0}\right)=V_{D}^{0}$ and $\frac{d V_{S}\left(t_{0}\right)}{d t}=r_{D}^{0}$ it takes place from Equations $(1.1-3)$, 


$$
\begin{aligned}
& V_{S}(t)=\lambda_{S} \frac{C_{3}}{k_{1}} \exp \left\{k_{1}\left(t-t_{0}\right)\right\}+\lambda_{S} \frac{C_{4}}{k_{2}} \exp \left\{k_{2}\left(t-t_{0}\right)\right\}+\left(r_{D}^{0}+\delta_{R}-\frac{\lambda_{D}}{\lambda_{S}} \varepsilon_{R}\right)\left(t-t_{0}\right) \\
& +\frac{\varepsilon_{R}}{2}\left(t-t_{0}\right)^{2}+V_{D}^{0}-\frac{\lambda_{D}}{\lambda_{S}} \delta_{R}-\frac{1}{\lambda_{P} \lambda_{S}} \varepsilon_{R}+\frac{\lambda_{D}^{2}}{\lambda_{S}^{2}} \varepsilon_{R}
\end{aligned}
$$

and it follows from Equations (1.60), (1.68), (1.72) for $t \rightarrow+\infty$,

$$
\begin{aligned}
& V_{S}(t) \rightarrow\left(r_{D}^{0}+\delta_{R}-\frac{\lambda_{D}}{\lambda_{S}} \varepsilon_{R}\right)\left(t-t_{0}\right)+\frac{\varepsilon_{R}}{2}\left(t-t_{0}\right)^{2}+V_{D}^{0}-\frac{\lambda_{D}}{\lambda_{S}} \delta_{R}-\frac{1}{\lambda_{P} \lambda_{S}} \varepsilon_{R}+\frac{\lambda_{D}^{2}}{\lambda_{S}^{2}} \varepsilon_{R} \\
& V_{D}(t) \rightarrow\left(r_{D}^{0}-\frac{\lambda_{D}}{\lambda_{S}} \varepsilon_{R}\right)\left(t-t_{0}\right)+V_{D}^{0}-\frac{\lambda_{D}}{\lambda_{S}} \delta_{R}+\frac{\lambda_{D}^{2}}{\lambda_{S}^{2}} \varepsilon_{R} \\
& S_{R}(t)=\delta_{R}\left(t-t_{0}\right)+\frac{\varepsilon_{R}}{2}\left(t-t_{0}\right)^{2}
\end{aligned}
$$

Since $\lim _{t \rightarrow+\infty}\left(\frac{P_{R}(t)}{\widetilde{P}_{R}(t)}\right)=+\infty \quad\left(\right.$ as $\left.\frac{P_{R}(t)}{\widetilde{P}_{R}(t)} \rightarrow \frac{\varepsilon_{R}}{\lambda_{S} P_{R}^{0}}\left(t-t_{0}\right)+\left(1+\frac{\delta_{R}}{\lambda_{S} P_{R}^{0}}-\frac{\lambda_{D} \varepsilon_{R}}{\lambda_{S}^{2} P_{R}^{0}}\right), t \rightarrow+\infty\right)$

and $\lim _{t \rightarrow+\infty}\left(\frac{V_{D}(t)}{\widetilde{V}_{D}(t)}\right)=1-\frac{\lambda_{D} \varepsilon_{R}}{\lambda_{S} r_{D}^{0}}$, it takes place,

$$
\lim _{t \rightarrow+\infty}\left(\frac{v_{R}(t)}{\widetilde{v}_{R}(t)}\right)=\lim _{t \rightarrow+\infty}\left(\frac{P_{R}(t) \times V_{D}(t)}{\widetilde{P}_{R}(t) \times \widetilde{V}_{D}(t)}\right)=\left\{\begin{array}{cc}
+\infty, & \varepsilon_{R}<\frac{\lambda_{S}}{\lambda_{D}} r_{D}^{0} \\
0, & \varepsilon_{R}=\frac{\lambda_{S}}{\lambda_{D}} r_{D}^{0} \\
-\infty, & \varepsilon_{R}>\frac{\lambda_{S}}{\lambda_{D}} r_{D}^{0}
\end{array}\right.
$$

(b) If $\frac{\lambda_{P}^{2} \lambda_{D}^{2}}{4}=\lambda_{P} \lambda_{S}$ the solution of Equation (1.62) is,

$$
D_{1}(t)=\left(C_{1}+C_{2}\left(t-t_{0}\right)\right) \exp \left\{-\frac{\lambda_{P} \lambda_{D}}{2}\left(t-t_{0}\right)\right\}
$$

where $C_{1}=\frac{1}{\lambda_{P} \lambda_{S}} \varepsilon_{R}$ and $C_{2}=-\delta_{R}+\frac{\lambda_{D}}{2 \lambda_{S}} \varepsilon_{R}$. 
If equality above holds, the solution of Equation (1.64) is

$$
P_{1}(t)=\left(C_{3}+C_{4}\left(t-t_{0}\right)\right) \exp \left\{-\frac{\lambda_{P} \lambda_{D}}{2}\left(t-t_{0}\right)\right\},
$$

where $C_{3}=-\frac{1}{\lambda_{S}} \delta_{R}+\frac{\lambda_{D}}{\lambda_{S}^{2}} \varepsilon_{R}$ and $C_{4}=-\frac{2}{\lambda_{D}} \delta_{R}+\frac{1}{\lambda_{S}} \varepsilon_{R}$ are constants.

Since $\lambda_{P} \lambda_{D}>0$ it takes place $D_{1}(t) \rightarrow 0$ and $P_{1}(t) \rightarrow 0$ for $t \rightarrow+\infty$.

Then it follows from the change of variable,

$$
\begin{aligned}
& D_{R}(t)=D_{1}(t)-\frac{1}{\lambda_{P} \lambda_{S}} \varepsilon_{R} \\
& P_{R}(t)=P_{1}(t)+P_{R}^{0}+\frac{1}{\lambda_{S}} \delta_{R}+\frac{1}{\lambda_{S}} \varepsilon_{R}\left(t-t_{0}\right)-\frac{\lambda_{D}}{\lambda_{S}^{2}} \varepsilon_{R}
\end{aligned}
$$

and it takes place for $t \rightarrow+\infty$,

$$
\begin{aligned}
& D_{R}(t) \rightarrow-\frac{1}{\lambda_{P} \lambda_{S}} \varepsilon_{R} \\
& P_{R}(t) \rightarrow \frac{1}{\lambda_{S}} \varepsilon_{R}\left(t-t_{0}\right)+P_{R}^{0}+\frac{1}{\lambda_{S}} \delta_{R}-\frac{\lambda_{D}}{\lambda_{S}^{2}} \varepsilon_{R}
\end{aligned}
$$

Since $V_{S}\left(t_{0}\right)=V_{D}^{0}$ and $\frac{d V_{S}\left(t_{0}\right)}{d t}=r_{D}^{0}$ it takes place from Equations $(1.1-3)$,

$$
\begin{aligned}
& V_{S}(t)=\left(-\frac{\lambda_{D}}{2} C_{3}-\frac{1}{\lambda_{P}} C_{4}\left(\frac{\lambda_{P} \lambda_{D}}{2}\left(t-t_{0}\right)+1\right)\right) \exp \left\{-\frac{\lambda_{P} \lambda_{D}}{2}\left(t-t_{0}\right)\right\}+\left(r_{D}^{0}+\delta_{R}-\frac{\lambda_{D}}{\lambda_{S}} \varepsilon_{R}\right)\left(t-t_{0}\right) \\
& +\frac{\varepsilon_{R}}{2}\left(t-t_{0}\right)^{2}+V_{D}^{0}-\frac{\lambda_{D}}{\lambda_{S}} \delta_{R}+\frac{3}{\lambda_{P} \lambda_{S}} \varepsilon_{R}
\end{aligned}
$$

and it follows from Equations (1.60), (1.81), (1.83) for $t \rightarrow+\infty$,

$$
\begin{aligned}
& V_{S}(t) \rightarrow\left(r_{D}^{0}+\delta_{R}-\frac{\lambda_{D}}{\lambda_{S}} \varepsilon_{R}\right)\left(t-t_{0}\right)+\frac{\varepsilon_{R}}{2}\left(t-t_{0}\right)^{2}+V_{D}^{0}-\frac{\lambda_{D}}{\lambda_{S}} \delta_{R}+\frac{3}{\lambda_{P} \lambda_{S}} \varepsilon_{R} \\
& V_{D}(t) \rightarrow\left(r_{D}^{0}-\frac{\lambda_{D}}{\lambda_{S}} \varepsilon_{R}\right)\left(t-t_{0}\right)+V_{D}^{0}-\frac{\lambda_{D}}{\lambda_{S}} \delta_{R}+\frac{4}{\lambda_{P} \lambda_{S}} \varepsilon_{R}
\end{aligned}
$$


$S_{R}(t)=\delta_{R}\left(t-t_{0}\right)+\frac{\varepsilon_{R}}{2}\left(t-t_{0}\right)^{2}$

Since $\lim _{t \rightarrow+\infty}\left(\frac{P_{R}(t)}{\widetilde{P}_{R}(t)}\right)=+\infty \quad\left(\right.$ as $\left.\frac{P_{R}(t)}{\widetilde{P}_{R}(t)} \rightarrow \frac{\varepsilon_{R}}{\lambda_{S} P_{R}^{0}}\left(t-t_{0}\right)+\left(1+\frac{\delta_{R}}{\lambda_{S} P_{R}^{0}}-\frac{\lambda_{D} \varepsilon_{R}}{\lambda_{S}^{2} P_{R}^{0}}\right), t \rightarrow+\infty\right)$

and $\lim _{t \rightarrow+\infty}\left(\frac{V_{D}(t)}{\widetilde{V}_{D}(t)}\right)=1-\frac{\lambda_{D} \varepsilon_{R}}{\lambda_{S} r_{D}^{0}}$, it takes place,

$$
\lim _{t \rightarrow+\infty}\left(\frac{v_{R}(t)}{\widetilde{v}_{R}(t)}\right)=\lim _{t \rightarrow+\infty}\left(\frac{P_{R}(t) \times V_{D}(t)}{\widetilde{P}_{R}(t) \times \widetilde{V}_{D}(t)}\right)=\left\{\begin{array}{cc}
+\infty, & \varepsilon_{R}<\frac{\lambda_{S}}{\lambda_{D}} r_{D}^{0} \\
0, & \varepsilon_{R}=\frac{\lambda_{S}}{\lambda_{D}} r_{D}^{0} \\
-\infty, & \varepsilon_{R}>\frac{\lambda_{S}}{\lambda_{D}} r_{D}^{0}
\end{array}\right.
$$

(c) If $\frac{\lambda_{P}^{2} \lambda_{D}^{2}}{4}<\lambda_{P} \lambda_{S}$ the solution of Equation (1.62) is,

$D_{1}(t)=\exp \left\{-\frac{\lambda_{P} \lambda_{D}}{2}\left(t-t_{0}\right)\right\}\left(C_{1} \cos \left(\sqrt{\lambda_{P} \lambda_{S}-\frac{\lambda_{P}^{2} \lambda_{D}^{2}}{4}}\left(t-t_{0}\right)\right)+C_{2} \sin \left(\sqrt{\lambda_{P} \lambda_{S}-\frac{\lambda_{P}^{2} \lambda_{D}^{2}}{4}}\left(t-t_{0}\right)\right)\right)$

where $C_{1}=\frac{1}{\lambda_{P} \lambda_{S}} \varepsilon_{R}$ and $C_{2}=\frac{-\delta_{R}+\frac{\lambda_{D}}{2 \lambda_{S}} \varepsilon_{R}}{\sqrt{\lambda_{P} \lambda_{S}-\frac{\lambda_{P}^{2} \lambda_{D}^{2}}{4}}}$

If inequality above holds, the solution of Equation (1.64) is

$$
P_{1}(t)=\exp \left\{-\frac{\lambda_{P} \lambda_{D}}{2}\left(t-t_{0}\right)\right\}\left(C_{3} \cos \left(\sqrt{\lambda_{P} \lambda_{S}-\frac{\lambda_{P}^{2} \lambda_{D}^{2}}{4}}\left(t-t_{0}\right)\right)+C_{4} \sin \left(\sqrt{\lambda_{P} \lambda_{S}-\frac{\lambda_{P}^{2} \lambda_{D}^{2}}{4}}\left(t-t_{0}\right)\right)\right)
$$

where $C_{3}=-\frac{1}{\lambda_{S}} \delta_{R}+\frac{\lambda_{D}}{\lambda_{S}^{2}} \varepsilon_{R}$ and $C_{4}=\frac{-\frac{\lambda_{P} \lambda_{D}}{2 \lambda_{S}} \delta_{R}+\frac{\lambda_{P} \lambda_{D}^{2}}{2 \lambda_{S}^{2}} \varepsilon_{R}-\frac{1}{\lambda_{S}} \varepsilon_{R}}{\sqrt{\lambda_{P} \lambda_{S}-\frac{\lambda_{P}^{2} \lambda_{D}^{2}}{4}}}$ are constants.

Since $\lambda_{P} \lambda_{D}>0$ it takes place $D_{1}(t) \rightarrow 0$ and $P_{1}(t) \rightarrow 0$ for $t \rightarrow+\infty$. 
Then it follows from the change of variable,

$$
\begin{aligned}
& D_{R}(t)=D_{1}(t)-\frac{1}{\lambda_{P} \lambda_{S}} \varepsilon_{R} \\
& P_{R}(t)=P_{1}(t)+P_{R}^{0}+\frac{1}{\lambda_{S}} \delta_{R}+\frac{1}{\lambda_{S}} \varepsilon_{R}\left(t-t_{0}\right)-\frac{\lambda_{D}}{\lambda_{S}^{2}} \varepsilon_{R}
\end{aligned}
$$

and it takes place for $t \rightarrow+\infty$,

$$
\begin{aligned}
& D_{R}(t) \rightarrow-\frac{1}{\lambda_{P} \lambda_{S}} \varepsilon_{R} \\
& P_{R}(t) \rightarrow \frac{1}{\lambda_{S}} \varepsilon_{R}\left(t-t_{0}\right)+P_{R}^{0}+\frac{1}{\lambda_{S}} \delta_{R}-\frac{\lambda_{D}}{\lambda_{S}^{2}} \varepsilon_{R}
\end{aligned}
$$

Since $V_{S}\left(t_{0}\right)=V_{D}^{0}$ and $\frac{d V_{S}\left(t_{0}\right)}{d t}=r_{D}^{0}$ it takes place from Equations $(1.1-3)$,

$$
\begin{aligned}
& V_{S}(t)=\frac{1}{\lambda_{P}} \cdot \exp \left\{-\frac{\lambda_{P} \lambda_{D}}{2}\left(t-t_{0}\right)\right\} \times\left(\begin{array}{l}
-\left(C_{3} \cdot \frac{\lambda_{P} \lambda_{D}}{2}+C_{4} \cdot \sqrt{\lambda_{P} \lambda_{S}-\frac{\lambda_{P}^{2} \lambda_{D}^{2}}{4}}\right) \cdot \cos \left(\sqrt{\lambda_{P} \lambda_{S}-\frac{\lambda_{P}^{2} \lambda_{D}^{2}}{4}}\left(t-t_{0}\right)\right. \\
+\left(C_{3} \cdot \sqrt{\lambda_{P} \lambda_{S}-\frac{\lambda_{P}^{2} \lambda_{D}^{2}}{4}}-C_{4} \cdot \frac{\lambda_{P} \lambda_{D}}{2}\right) \cdot \sin \left(\sqrt{\lambda_{P} \lambda_{S}-\frac{\lambda_{P}^{2} \lambda_{D}^{2}}{4}}\left(t-t_{0}\right)\right)
\end{array}\right) \\
& +\left(r_{D}^{0}+\delta_{R}-\frac{\lambda_{D}}{\lambda_{S}} \varepsilon_{R}\right)\left(t-t_{0}\right)+\frac{\varepsilon_{R}}{2}\left(t-t_{0}\right)^{2}+V_{D}^{0}-\frac{\lambda_{D}}{\lambda_{S}} \delta_{R}-\frac{1}{\lambda_{P} \lambda_{S}} \varepsilon_{R}+\frac{\lambda_{D}^{2}}{\lambda_{S}^{2}} \varepsilon_{R}
\end{aligned}
$$

and it follows from Equations (1.60), (1.92), (1.94) for $t \rightarrow+\infty$,

$$
\begin{aligned}
& V_{S}(t) \rightarrow\left(r_{D}^{0}+\delta_{R}-\frac{\lambda_{D}}{\lambda_{S}} \varepsilon_{R}\right)\left(t-t_{0}\right)+\frac{\varepsilon_{R}}{2}\left(t-t_{0}\right)^{2}+V_{D}^{0}-\frac{\lambda_{D}}{\lambda_{S}} \delta_{R}-\frac{1}{\lambda_{P} \lambda_{S}} \varepsilon_{R}+\frac{\lambda_{D}^{2}}{\lambda_{S}^{2}} \varepsilon_{R} \\
& V_{D}(t) \rightarrow\left(r_{D}^{0}-\frac{\lambda_{D}}{\lambda_{S}} \varepsilon_{R}\right)\left(t-t_{0}\right)+V_{D}^{0}-\frac{\lambda_{D}}{\lambda_{S}} \delta_{R}+\frac{\lambda_{D}^{2}}{\lambda_{S}^{2}} \varepsilon_{R} \\
& S_{R}(t)=\delta_{R}\left(t-t_{0}\right)+\frac{\varepsilon_{R}}{2}\left(t-t_{0}\right)^{2}
\end{aligned}
$$


Since $\lim _{t \rightarrow+\infty}\left(\frac{P_{R}(t)}{\widetilde{P}_{R}(t)}\right)=+\infty \quad\left(\right.$ as $\left.\frac{P_{R}(t)}{\widetilde{P}_{R}(t)} \rightarrow \frac{\varepsilon_{R}}{\lambda_{S} P_{R}^{0}}\left(t-t_{0}\right)+\left(1+\frac{\delta_{R}}{\lambda_{S} P_{R}^{0}}-\frac{\lambda_{D} \varepsilon_{R}}{\lambda_{S}^{2} P_{R}^{0}}\right), t \rightarrow+\infty\right)$ and $\lim _{t \rightarrow+\infty}\left(\frac{V_{D}(t)}{\widetilde{V}_{D}(t)}\right)=1-\frac{\lambda_{D} \varepsilon_{R}}{\lambda_{S} r_{D}^{0}}$, it takes place,

$$
\lim _{t \rightarrow+\infty}\left(\frac{v_{R}(t)}{\widetilde{v}_{R}(t)}\right)=\lim _{t \rightarrow+\infty}\left(\frac{P_{R}(t) \times V_{D}(t)}{\widetilde{P}_{R}(t) \times \widetilde{V}_{D}(t)}\right)=\left\{\begin{array}{cc}
+\infty, & \varepsilon_{R}<\frac{\lambda_{S}}{\lambda_{D}} r_{D}^{0} \\
0, & \varepsilon_{R}=\frac{\lambda_{S}}{\lambda_{D}} r_{D}^{0} \\
-\infty, & \varepsilon_{R}>\frac{\lambda_{S}}{\lambda_{D}} r_{D}^{0}
\end{array}\right.
$$

Thus all cases to deduce the solutions of differential equations from the roots of related characteristic equation are covered.

Therefore at the limit for $t \rightarrow+\infty$ the withdrawal of product from the market causes a boundless increase of the product's price. Here at the limit for $t \rightarrow+\infty$ the withdrawal of product from the market increases or decreases the product's demand depending on the acceleration rate. Savings withdrawal with moderate acceleration rate increases the product demand on the market in the long run. Savings withdrawal with intense acceleration rate decreases the product demand on the market in the long run. Thus savings withdrawal with moderate acceleration rate raises the monetary value $v_{R}(t)$ of product on the market in the long run, and savings withdrawal with intense acceleration rate declines the product demand on the market in the long run,

$$
\begin{aligned}
& \lim _{t \rightarrow+\infty}\left(\frac{P_{R}(t)}{\widetilde{P}_{R}(t)}\right)=+\infty, \\
& \lim _{t \rightarrow+\infty}\left(\frac{V_{D}(t)}{\widetilde{V}_{D}(t)}\right)=1-\frac{\lambda_{D} \varepsilon_{R}}{\lambda_{S} r_{D}^{0}} \begin{cases}>0, & \varepsilon_{R}<\frac{\lambda_{S}}{\lambda_{D}} r_{D}^{0} \\
=0, & \varepsilon_{R}=\frac{\lambda_{S}}{\lambda_{D}} r_{D}^{0}, \\
<0, & \varepsilon_{R}>\frac{\lambda_{S}}{\lambda_{D}} r_{D}^{0}\end{cases}
\end{aligned}
$$




$$
\lim _{t \rightarrow+\infty}\left(\frac{v_{R}(t)}{\widetilde{v}_{R}(t)}\right)=\lim _{t \rightarrow+\infty}\left(\frac{P_{R}(t)}{\widetilde{P}_{R}(t)} \times \frac{V_{D}(t)}{\widetilde{V}_{D}(t)}\right)=\left\{\begin{array}{cc}
+\infty, & \varepsilon_{R}<\frac{\lambda_{S}}{\lambda_{D}} r_{D}^{0} \\
0, & \varepsilon_{R}=\frac{\lambda_{S}}{\lambda_{D}} r_{D}^{0} \\
-\infty, & \varepsilon_{R}>\frac{\lambda_{S}}{\lambda_{D}} r_{D}^{0}
\end{array}\right.
$$

That concludes the third scenario.

\section{Exponential Continuous Withdrawal of Product Savings from Market}

According to this scenario I assume that amount of product's savings $S_{R}(t)$ increases since time $t=t_{0}$ according to following formula,

$$
S_{R}(t)=\left\{\begin{array}{cc}
0, & t<t_{0} \\
\pi \exp \left\{\rho_{R}\left(t-t_{0}\right)\right\}-\pi, & t \geq t_{0}
\end{array}\right.
$$

where $S_{R}(t)=0$ for $t<t_{0}, \pi>0$, and $\rho_{R}>0$.

Therefore taken into account the product's withdrawal from the market in the form of product's savings $S_{R}(t)$ described by Equation (1.60) the volume of product's surplus (or shortage) on the market $\left[V_{S}(t)-V_{D}(t)\right]$ in Equations $(1.1-3)$ has to be replaced by the volume of product's surplus (or shortage) on the market expressed as $D_{R} \equiv\left(V_{S}(t)-V_{D}(t)-S_{R}(t)\right)$; that produces for $t>t_{0}$,

$$
\frac{d^{2} D_{R}(t)}{d t^{2}}+\lambda_{P} \lambda_{D} \frac{d D_{R}(t)}{d t}+\lambda_{P} \lambda_{S} D_{R}(t)+\pi \rho_{R}^{2} \exp \left\{\rho_{R}\left(t-t_{0}\right)\right\}=0
$$

with the following initial conditions,

$$
\begin{aligned}
& D_{R}\left(t_{0}\right)=0, \\
& \frac{d D_{R}\left(t_{0}\right)}{d t}=-\pi \rho_{R} .
\end{aligned}
$$


If one uses the new variable $D_{1}(t)=D_{R}(t)+\frac{\pi \rho_{R}^{2}}{\rho_{R}^{2}+\lambda_{P} \lambda_{D} \rho_{R}+\lambda_{P} \lambda_{S}} \exp \left\{\rho_{R}\left(t-t_{0}\right)\right\}$, then Equation (1.100) becomes,

$$
\frac{d^{2} D_{1}(t)}{d t^{2}}+\lambda_{P} \lambda_{D} \frac{d D_{1}(t)}{d t}+\lambda_{P} \lambda_{S} D_{1}(t)=0
$$

with the following initial conditions,

$$
\begin{aligned}
& D_{1}\left(t_{0}\right)=\frac{\pi \rho_{R}^{2}}{\rho_{R}^{2}+\lambda_{P} \lambda_{D} \rho_{R}+\lambda_{P} \lambda_{S}}, \\
& \frac{d D_{1}\left(t_{0}\right)}{d t}=-\pi \rho_{R}+\frac{\pi \rho_{R}^{3}}{\rho_{R}^{2}+\lambda_{P} \lambda_{D} \rho_{R}+\lambda_{P} \lambda_{S}} .
\end{aligned}
$$

Initial conditions for the product's price $P_{R}(t)$ are $P_{R}\left(t_{0}\right)=P_{R}^{0}$ and $\frac{d P_{R}\left(t_{0}\right)}{d t}=0$

Similar to Equation (1.100) the product's price $P_{R}(t)$ is described by the following second-order ordinary differential equation for $t>t_{0}$,

$$
\frac{d^{2} P_{R}(t)}{d t^{2}}+\lambda_{P} \lambda_{D} \frac{d P_{R}(t)}{d t}+\lambda_{P} \lambda_{S} P_{R}(t)-\lambda_{P}\left(\pi \rho_{R} \exp \left\{\rho_{R}\left(t-t_{0}\right)\right\}+\lambda_{S} P_{R}^{0}\right)=0
$$

If one uses the new variable $P_{1}(t)=P_{R}(t)-P_{R}^{0}-\frac{\lambda_{P} \pi \rho_{R}}{\rho_{R}^{2}+\lambda_{P} \lambda_{D} \rho_{R}+\lambda_{P} \lambda_{S}} \exp \left\{\rho_{R}\left(t-t_{0}\right)\right\}$, then Equation (1.102) becomes,

$$
\frac{d^{2} P_{1}(t)}{d t^{2}}+\lambda_{P} \lambda_{D} \frac{d P_{1}(t)}{d t}+\lambda_{P} \lambda_{S} P_{1}(t)=0
$$

Therefore initial conditions for $P_{1}(t)$ are $P_{1}\left(t_{0}\right)=\frac{-\lambda_{P} \pi \rho_{R}}{\rho_{R}^{2}+\lambda_{P} \lambda_{D} \rho_{R}+\lambda_{P} \lambda_{S}} \quad$ and $\frac{d P_{1}\left(t_{0}\right)}{d t}=\frac{-\lambda_{P} \pi \rho_{R}^{2}}{\rho_{R}^{2}+\lambda_{P} \lambda_{D} \rho_{R}+\lambda_{P} \lambda_{S}}$.

Equations (1.101) and (1.103) have the same characteristic equations. And the roots of these characteristic equations are, 


$$
k_{1,2}=-\frac{\lambda_{P} \lambda_{D}}{2} \pm \sqrt{\frac{\lambda_{P}^{2} \lambda_{D}^{2}}{4}-\lambda_{P} \lambda_{S}}
$$

(a) If $\frac{\lambda_{P}^{2} \lambda_{D}^{2}}{4}>\lambda_{P} \lambda_{S}$ the solution of Equation (1.101) is,

$$
D_{1}(t)=C_{1} \exp \left\{k_{1}\left(t-t_{0}\right)\right\}+C_{2} \exp \left\{k_{2}\left(t-t_{0}\right)\right\}
$$

where constants $C_{1}$ and $C_{2}$ are equal to $C_{1}=-\frac{\pi \rho_{R}}{k_{1}-k_{2}}+\frac{1-k_{2}}{k_{1}-k_{2}} \cdot \frac{\pi \rho_{R}^{2}}{\rho_{R}^{2}+\lambda_{P} \lambda_{D} \rho_{R}+\lambda_{P} \lambda_{S}}$ and $C_{2}=-\frac{\pi \rho_{R}}{k_{2}-k_{1}}+\frac{1-k_{1}}{k_{2}-k_{1}} \cdot \frac{\pi \rho_{R}^{2}}{\rho_{R}^{2}+\lambda_{P} \lambda_{D} \rho_{R}+\lambda_{P} \lambda_{S}}$.

If inequality above holds, the solution of Equation (1.103) is

$$
P_{1}(t)=C_{3} \exp \left\{k_{1}\left(t-t_{0}\right)\right\}+C_{4} \exp \left\{k_{2}\left(t-t_{0}\right)\right\}
$$

where $C_{3}=\frac{\rho_{R}-k_{2}}{k_{2}-k_{1}} \cdot \frac{\lambda_{P} \pi \rho_{R}}{\rho_{R}^{2}+\lambda_{P} \lambda_{D} \rho_{R}+\lambda_{P} \lambda_{S}}$ and $C_{4}=\frac{\rho_{R}-k_{1}}{k_{1}-k_{2}} \cdot \frac{\lambda_{P} \pi \rho_{R}}{\rho_{R}^{2}+\lambda_{P} \lambda_{D} \rho_{R}+\lambda_{P} \lambda_{S}}$ are constants.

Since $k_{1}<0$ and $k_{2}<0$ it takes place $D_{1}(t) \rightarrow 0$ and $P_{1}(t) \rightarrow 0$ for $t \rightarrow+\infty$.

Then it follows from the change of variable,

$$
\begin{aligned}
& D_{R}(t)=D_{1}(t)-\frac{\pi \rho_{R}^{2}}{\rho_{R}^{2}+\lambda_{P} \lambda_{D} \rho_{R}+\lambda_{P} \lambda_{S}} \exp \left\{\rho_{R}\left(t-t_{0}\right)\right\} \\
& P_{R}(t)=P_{1}(t)+P_{R}^{0}+\frac{\lambda_{P} \pi \rho_{R}}{\rho_{R}^{2}+\lambda_{P} \lambda_{D} \rho_{R}+\lambda_{P} \lambda_{S}} \exp \left\{\rho_{R}\left(t-t_{0}\right)\right\}
\end{aligned}
$$

and it takes place for $t \rightarrow+\infty$,

$$
\begin{aligned}
& D_{R}(t) \rightarrow \frac{-\pi \rho_{R}^{2}}{\rho_{R}^{2}+\lambda_{P} \lambda_{D} \rho_{R}+\lambda_{P} \lambda_{S}} \exp \left\{\rho_{R}\left(t-t_{0}\right)\right\} \\
& P_{R}(t) \rightarrow P_{R}^{0}+\frac{\lambda_{P} \pi \rho_{R}}{\rho_{R}^{2}+\lambda_{P} \lambda_{D} \rho_{R}+\lambda_{P} \lambda_{S}} \exp \left\{\rho_{R}\left(t-t_{0}\right)\right\}
\end{aligned}
$$

Since $V_{S}\left(t_{0}\right)=V_{D}^{0}$ and $\frac{d V_{S}\left(t_{0}\right)}{d t}=r_{D}^{0}$ it takes place from Equations $(1.1-3)$, 


$$
\begin{aligned}
& V_{S}(t)=\lambda_{S} \frac{C_{3}}{k_{1}} \exp \left\{k_{1}\left(t-t_{0}\right)\right\}+\lambda_{S} \frac{C_{4}}{k_{2}} \exp \left\{k_{2}\left(t-t_{0}\right)\right\}+r_{D}^{0}\left(t-t_{0}\right)+V_{D}^{0} \\
& +\frac{\lambda_{P} \lambda_{S} \pi}{\rho_{R}^{2}+\lambda_{P} \lambda_{D} \rho_{R}+\lambda_{P} \lambda_{S}} \exp \left\{\rho_{R}\left(t-t_{0}\right)\right\}-\pi
\end{aligned}
$$

and it follows from Equations (1.99), (1.109), (1.111) for $t \rightarrow+\infty$,

$$
\begin{aligned}
& V_{S}(t) \rightarrow r_{D}^{0}\left(t-t_{0}\right)+V_{D}^{0}+\frac{\lambda_{P} \lambda_{S} \pi}{\rho_{R}^{2}+\lambda_{P} \lambda_{D} \rho_{R}+\lambda_{P} \lambda_{S}} \exp \left\{\rho_{R}\left(t-t_{0}\right)\right\}-\pi \\
& V_{D}(t) \rightarrow r_{D}^{0}\left(t-t_{0}\right)+V_{D}^{0}-\frac{\lambda_{P} \lambda_{D} \pi \rho_{R}}{\rho_{R}^{2}+\lambda_{P} \lambda_{D} \rho_{R}+\lambda_{P} \lambda_{S}} \exp \left\{\rho_{R}\left(t-t_{0}\right)\right\} \\
& S_{R}(t)=\pi \exp \left\{\rho_{R}\left(t-t_{0}\right)\right\}-\pi
\end{aligned}
$$

Since $\lim _{t \rightarrow+\infty}\left(\frac{P_{R}(t)}{\widetilde{P}_{R}(t)}\right)=+\infty \quad\left(\right.$ as $\left.\frac{P_{R}(t)}{\widetilde{P}_{R}(t)} \rightarrow \frac{\lambda_{P} \pi \rho_{R}}{\left(\rho_{R}^{2}+\lambda_{P} \lambda_{D} \rho_{R}+\lambda_{P} \lambda_{S}\right) P_{R}^{0}} \exp \left\{\rho_{R}\left(t-t_{0}\right)\right\}+1\right)$

and $\lim _{t \rightarrow+\infty}\left(\frac{V_{D}(t)}{\widetilde{V}_{D}(t)}\right)=-\infty \quad$ as $\quad \frac{V_{D}(t)}{\widetilde{V}_{D}(t)} \rightarrow \frac{-\lambda_{P} \lambda_{D} \pi \rho_{R}}{\left(\rho_{R}^{2}+\lambda_{P} \lambda_{D} \rho_{R}+\lambda_{P} \lambda_{S}\right) r_{D}^{0}} \cdot \frac{\exp \left\{\rho_{R}\left(t-t_{0}\right)\right\}}{\left(t-t_{0}\right)}+1$ when $t \rightarrow+\infty)$, it takes place,

$$
\lim _{t \rightarrow+\infty}\left(\frac{v_{R}(t)}{\widetilde{v}_{R}(t)}\right)=\lim _{t \rightarrow+\infty}\left(\frac{P_{R}(t) \times V_{D}(t)}{\widetilde{P}_{R}(t) \times \widetilde{V}_{D}(t)}\right)=-\infty
$$

(b) If $\frac{\lambda_{P}^{2} \lambda_{D}^{2}}{4}=\lambda_{P} \lambda_{S}$ the solution of Equation (1.101) is,

$$
D_{1}(t)=\left(C_{1}+C_{2}\left(t-t_{0}\right)\right) \exp \left\{-\frac{\lambda_{P} \lambda_{D}}{2}\left(t-t_{0}\right)\right\}
$$

where $C_{1}=\frac{\pi \rho_{R}^{2}}{\rho_{R}^{2}+\lambda_{P} \lambda_{D} \rho_{R}+\lambda_{P} \lambda_{S}}$ and $C_{2}=\frac{-\lambda_{P} \pi \rho_{R}\left(2 \lambda_{S}+\lambda_{D} \rho_{R}\right)}{2\left(\rho_{R}^{2}+\lambda_{P} \lambda_{D} \rho_{R}+\lambda_{P} \lambda_{S}\right)}$.

If equality above holds, the solution of Equation (1.103) is

$$
P_{1}(t)=\left(C_{3}+C_{4}\left(t-t_{0}\right)\right) \exp \left\{-\frac{\lambda_{P} \lambda_{D}}{2}\left(t-t_{0}\right)\right\},
$$

where $C_{3}=\frac{-\lambda_{P} \pi \rho_{R}}{\rho_{R}^{2}+\lambda_{P} \lambda_{D} \rho_{R}+\lambda_{P} \lambda_{S}}$ and $C_{4}=\frac{-\lambda_{P} \pi \rho_{R}\left(2 \rho_{R}+\lambda_{P} \lambda_{D}\right)}{2\left(\rho_{R}^{2}+\lambda_{P} \lambda_{D} \rho_{R}+\lambda_{P} \lambda_{S}\right)}$ are constants. 
Since $\lambda_{P} \lambda_{D}>0$ it takes place $D_{1}(t) \rightarrow 0$ and $P_{1}(t) \rightarrow 0$ for $t \rightarrow+\infty$.

Then it follows from the change of variable,

$$
\begin{aligned}
& D_{R}(t)=D_{1}(t)-\frac{\pi \rho_{R}^{2}}{\rho_{R}^{2}+\lambda_{P} \lambda_{D} \rho_{R}+\lambda_{P} \lambda_{S}} \exp \left\{\rho_{R}\left(t-t_{0}\right)\right\} \\
& P_{R}(t)=P_{1}(t)+P_{R}^{0}+\frac{\lambda_{P} \pi \rho_{R}}{\rho_{R}^{2}+\lambda_{P} \lambda_{D} \rho_{R}+\lambda_{P} \lambda_{S}} \exp \left\{\rho_{R}\left(t-t_{0}\right)\right\}
\end{aligned}
$$

and it takes place for $t \rightarrow+\infty$,

$$
\begin{aligned}
& D_{R}(t) \rightarrow \frac{-\pi \rho_{R}^{2}}{\rho_{R}^{2}+\lambda_{P} \lambda_{D} \rho_{R}+\lambda_{P} \lambda_{S}} \exp \left\{\rho_{R}\left(t-t_{0}\right)\right\} \\
& P_{R}(t) \rightarrow P_{R}^{0}+\frac{\lambda_{P} \pi \rho_{R}}{\rho_{R}^{2}+\lambda_{P} \lambda_{D} \rho_{R}+\lambda_{P} \lambda_{S}} \exp \left\{\rho_{R}\left(t-t_{0}\right)\right\}
\end{aligned}
$$

Since $V_{S}\left(t_{0}\right)=V_{D}^{0}$ and $\frac{d V_{S}\left(t_{0}\right)}{d t}=r_{D}^{0}$ it takes place from Equations $(1.1-3)$,

$$
\begin{aligned}
& V_{S}(t)=\left(-\frac{\lambda_{D}}{2} C_{3}-\frac{1}{\lambda_{P}} C_{4}\left(\frac{\lambda_{P} \lambda_{D}}{2}\left(t-t_{0}\right)+1\right)\right) \exp \left\{-\frac{\lambda_{P} \lambda_{D}}{2}\left(t-t_{0}\right)\right\}+r_{D}^{0}\left(t-t_{0}\right) \\
& +V_{D}^{0}+\frac{\lambda_{P} \lambda_{S} \pi}{\rho_{R}^{2}+\lambda_{P} \lambda_{D} \rho_{R}+\lambda_{P} \lambda_{S}} \exp \left\{\rho_{R}\left(t-t_{0}\right)\right\}-\pi
\end{aligned}
$$

and it follows from Equations (1.99), (1.120), (1.122) for $t \rightarrow+\infty$,

$$
\begin{aligned}
& V_{S}(t) \rightarrow r_{D}^{0}\left(t-t_{0}\right)+V_{D}^{0}+\frac{\lambda_{P} \lambda_{S} \pi}{\rho_{R}^{2}+\lambda_{P} \lambda_{D} \rho_{R}+\lambda_{P} \lambda_{S}} \exp \left\{\rho_{R}\left(t-t_{0}\right)\right\}-\pi \\
& V_{D}(t) \rightarrow r_{D}^{0}\left(t-t_{0}\right)+V_{D}^{0}-\frac{\lambda_{P} \lambda_{D} \pi \rho_{R}}{\rho_{R}^{2}+\lambda_{P} \lambda_{D} \rho_{R}+\lambda_{P} \lambda_{S}} \exp \left\{\rho_{R}\left(t-t_{0}\right)\right\} \\
& S_{R}(t)=\pi \exp \left\{\rho_{R}\left(t-t_{0}\right)\right\}-\pi
\end{aligned}
$$

Since $\lim _{t \rightarrow+\infty}\left(\frac{P_{R}(t)}{\widetilde{P}_{R}(t)}\right)=+\infty \quad\left(\right.$ as $\left.\frac{P_{R}(t)}{\widetilde{P}_{R}(t)} \rightarrow \frac{\lambda_{P} \pi \rho_{R}}{\left(\rho_{R}^{2}+\lambda_{P} \lambda_{D} \rho_{R}+\lambda_{P} \lambda_{S}\right) P_{R}^{0}} \exp \left\{\rho_{R}\left(t-t_{0}\right)\right\}+1\right)$ and $\lim _{t \rightarrow+\infty}\left(\frac{V_{D}(t)}{\widetilde{V}_{D}(t)}\right)=-\infty \quad\left(\right.$ as $\quad \frac{V_{D}(t)}{\widetilde{V}_{D}(t)} \rightarrow \frac{-\lambda_{P} \lambda_{D} \pi \rho_{R}}{\left(\rho_{R}^{2}+\lambda_{P} \lambda_{D} \rho_{R}+\lambda_{P} \lambda_{S}\right) r_{D}^{0}} \cdot \frac{\exp \left\{\rho_{R}\left(t-t_{0}\right)\right\}}{\left(t-t_{0}\right)}+1$ when $t \rightarrow+\infty)$, it takes place, 


$$
\lim _{t \rightarrow+\infty}\left(\frac{v_{R}(t)}{\widetilde{v}_{R}(t)}\right)=\lim _{t \rightarrow+\infty}\left(\frac{P_{R}(t) \times V_{D}(t)}{\widetilde{P}_{R}(t) \times \widetilde{V}_{D}(t)}\right)=-\infty
$$

(c) If $\frac{\lambda_{P}^{2} \lambda_{D}^{2}}{4}<\lambda_{P} \lambda_{S}$ the solution of Equation (1.101) is,

$$
D_{1}(t)=\exp \left\{-\frac{\lambda_{P} \lambda_{D}}{2}\left(t-t_{0}\right)\right\}\left(C_{1} \cos \left(\sqrt{\lambda_{P} \lambda_{S}-\frac{\lambda_{P}^{2} \lambda_{D}^{2}}{4}}\left(t-t_{0}\right)\right)+C_{2} \sin \left(\sqrt{\lambda_{P} \lambda_{S}-\frac{\lambda_{P}^{2} \lambda_{D}^{2}}{4}}\left(t-t_{0}\right)\right)\right)
$$

where $C_{1}=\frac{\pi \rho_{R}^{2}}{\rho_{R}^{2}+\lambda_{P} \lambda_{D} \rho_{R}+\lambda_{P} \lambda_{S}}$ and $C_{2}=\frac{-\lambda_{P} \pi \rho_{R}\left(2 \lambda_{S}+\lambda_{D} \rho_{R}\right)}{2\left(\rho_{R}^{2}+\lambda_{P} \lambda_{D} \rho_{R}+\lambda_{P} \lambda_{S}\right)} \cdot \frac{1}{\sqrt{\lambda_{P} \lambda_{S}-\frac{\lambda_{P}^{2} \lambda_{D}^{2}}{4}}}$.

If inequality above holds, the solution of Equation (1.103) is

$$
P_{1}(t)=\exp \left\{-\frac{\lambda_{P} \lambda_{D}}{2}\left(t-t_{0}\right)\right\}\left(C_{3} \cos \left(\sqrt{\lambda_{P} \lambda_{S}-\frac{\lambda_{P}^{2} \lambda_{D}^{2}}{4}}\left(t-t_{0}\right)\right)+C_{4} \sin \left(\sqrt{\lambda_{P} \lambda_{S}-\frac{\lambda_{P}^{2} \lambda_{D}^{2}}{4}}\left(t-t_{0}\right)\right)\right)
$$

where $C_{3}=\frac{-\lambda_{P} \pi \rho_{R}}{\rho_{R}^{2}+\lambda_{P} \lambda_{D} \rho_{R}+\lambda_{P} \lambda_{S}}$ and $C_{4}=\frac{-\lambda_{P} \pi \rho_{R}\left(2 \rho_{R}+\lambda_{P} \lambda_{D}\right)}{2\left(\rho_{R}^{2}+\lambda_{P} \lambda_{D} \rho_{R}+\lambda_{P} \lambda_{S}\right)} \cdot \frac{1}{\sqrt{\lambda_{P} \lambda_{S}-\frac{\lambda_{P}^{2} \lambda_{D}^{2}}{4}}}$ are constants.

Since $\lambda_{P} \lambda_{D}>0$ it takes place $D_{1}(t) \rightarrow 0$ and $P_{1}(t) \rightarrow 0$ for $t \rightarrow+\infty$.

Then it follows from the change of variable,

$$
\begin{aligned}
& D_{R}(t)=D_{1}(t)-\frac{\pi \rho_{R}^{2}}{\rho_{R}^{2}+\lambda_{P} \lambda_{D} \rho_{R}+\lambda_{P} \lambda_{S}} \exp \left\{\rho_{R}\left(t-t_{0}\right)\right\} \\
& P_{R}(t)=P_{1}(t)+P_{R}^{0}+\frac{\lambda_{P} \pi \rho_{R}}{\rho_{R}^{2}+\lambda_{P} \lambda_{D} \rho_{R}+\lambda_{P} \lambda_{S}} \exp \left\{\rho_{R}\left(t-t_{0}\right)\right\}
\end{aligned}
$$

and it takes place for $t \rightarrow+\infty$,

$$
D_{R}(t) \rightarrow \frac{-\pi \rho_{R}^{2}}{\rho_{R}^{2}+\lambda_{P} \lambda_{D} \rho_{R}+\lambda_{P} \lambda_{S}} \exp \left\{\rho_{R}\left(t-t_{0}\right)\right\}
$$




$$
P_{R}(t) \rightarrow P_{R}^{0}+\frac{\lambda_{P} \pi \rho_{R}}{\rho_{R}^{2}+\lambda_{P} \lambda_{D} \rho_{R}+\lambda_{P} \lambda_{S}} \exp \left\{\rho_{R}\left(t-t_{0}\right)\right\}
$$

Since $V_{S}\left(t_{0}\right)=V_{D}^{0}$ and $\frac{d V_{S}\left(t_{0}\right)}{d t}=r_{D}^{0}$ it takes place from Equations $(1.1-3)$,

$$
\begin{aligned}
& V_{S}(t)=\frac{1}{\lambda_{P}} \cdot \exp \left\{-\frac{\lambda_{P} \lambda_{D}}{2}\left(t-t_{0}\right)\right\} \times\left(\begin{array}{l}
-\left(C_{3} \cdot \frac{\lambda_{P} \lambda_{D}}{2}+C_{4} \cdot \sqrt{\lambda_{P} \lambda_{S}-\frac{\lambda_{P}^{2} \lambda_{D}^{2}}{4}}\right) \cdot \cos \left(\sqrt{\lambda_{P} \lambda_{S}-\frac{\lambda_{P}^{2} \lambda_{D}^{2}}{4}}\left(t-t_{0}\right)\right. \\
+\left(C_{3} \cdot \sqrt{\lambda_{P} \lambda_{S}-\frac{\lambda_{P}^{2} \lambda_{D}^{2}}{4}}-C_{4} \cdot \frac{\lambda_{P} \lambda_{D}}{2}\right) \cdot \sin \left(\sqrt{\lambda_{P} \lambda_{S}-\frac{\lambda_{P}^{2} \lambda_{D}^{2}}{4}}\left(t-t_{0}\right)\right)
\end{array}\right) \\
& +r_{D}^{0}\left(t-t_{0}\right)+V_{D}^{0}+\frac{\lambda_{P} \lambda_{S} \pi}{\rho_{R}^{2}+\lambda_{P} \lambda_{D} \rho_{R}+\lambda_{P} \lambda_{S}} \exp \left\{\rho_{R}\left(t-t_{0}\right)\right\}-\pi
\end{aligned}
$$

and it follows from Equations (1.99), (1.131), (1.133) for $t \rightarrow+\infty$,

$$
\begin{aligned}
& V_{S}(t) \rightarrow r_{D}^{0}\left(t-t_{0}\right)+V_{D}^{0}+\frac{\lambda_{P} \lambda_{S} \pi}{\rho_{R}^{2}+\lambda_{P} \lambda_{D} \rho_{R}+\lambda_{P} \lambda_{S}} \exp \left\{\rho_{R}\left(t-t_{0}\right)\right\}-\pi \\
& V_{D}(t) \rightarrow r_{D}^{0}\left(t-t_{0}\right)+V_{D}^{0}-\frac{\lambda_{P} \lambda_{D} \pi \rho_{R}}{\rho_{R}^{2}+\lambda_{P} \lambda_{D} \rho_{R}+\lambda_{P} \lambda_{S}} \exp \left\{\rho_{R}\left(t-t_{0}\right)\right\} \\
& S_{R}(t)=\pi \exp \left\{\rho_{R}\left(t-t_{0}\right)\right\}-\pi
\end{aligned}
$$

Since $\lim _{t \rightarrow+\infty}\left(\frac{P_{R}(t)}{\widetilde{P}_{R}(t)}\right)=+\infty \quad\left(\right.$ as $\left.\frac{P_{R}(t)}{\widetilde{P}_{R}(t)} \rightarrow \frac{\lambda_{P} \pi \rho_{R}}{\left(\rho_{R}^{2}+\lambda_{P} \lambda_{D} \rho_{R}+\lambda_{P} \lambda_{S}\right) P_{R}^{0}} \exp \left\{\rho_{R}\left(t-t_{0}\right)\right\}+1\right)$

and $\lim _{t \rightarrow+\infty}\left(\frac{V_{D}(t)}{\widetilde{V}_{D}(t)}\right)=-\infty \quad$ as $\quad \frac{V_{D}(t)}{\widetilde{V}_{D}(t)} \rightarrow \frac{-\lambda_{P} \lambda_{D} \pi \rho_{R}}{\left(\rho_{R}^{2}+\lambda_{P} \lambda_{D} \rho_{R}+\lambda_{P} \lambda_{S}\right) r_{D}^{0}} \cdot \frac{\exp \left\{\rho_{R}\left(t-t_{0}\right)\right\}}{\left(t-t_{0}\right)}+1$ when $t \rightarrow+\infty)$, it takes place,

$$
\lim _{t \rightarrow+\infty}\left(\frac{v_{R}(t)}{\widetilde{v}_{R}(t)}\right)=\lim _{t \rightarrow+\infty}\left(\frac{P_{R}(t) \times V_{D}(t)}{\widetilde{P}_{R}(t) \times \widetilde{V}_{D}(t)}\right)=-\infty
$$

Thus all cases to deduce the solutions of differential equations from the roots of related characteristic equation are covered.

Therefore at the limit for $t \rightarrow+\infty$ the withdrawal of product from the market causes a boundless increase of the product's price. Here at the limit for $t \rightarrow+\infty$ the withdrawal of product from the market 
causes a boundless decrease of the product's demand. These actions causes a boundless decrease of the monetary value $v_{R}(t)$ of product on the market in the long run,

$$
\begin{aligned}
& \lim _{t \rightarrow+\infty}\left(\frac{P_{R}(t)}{\widetilde{P}_{R}(t)}=+\infty,\right. \\
& \lim _{t \rightarrow+\infty}\left(\frac{V_{D}(t)}{\widetilde{V}_{D}(t)}\right)=-\infty, \\
& \lim _{t \rightarrow+\infty}\left(\frac{v_{R}(t)}{\widetilde{v}_{R}(t)}\right)=\lim _{t \rightarrow+\infty}\left(\frac{P_{R}(t)}{\widetilde{P}_{R}(t)} \times \frac{V_{D}(t)}{\widetilde{V}_{D}(t)}\right)=-\infty
\end{aligned}
$$

That concludes the forth scenario.

\section{Model of Multiple Product Economy}

Let me consider model with $n$ products. I denote $\bar{V}_{S}(t) \geq 0$ as a non-negative vector of the products' supply on the market, $\bar{V}_{D}(t) \geq 0$ as a non-negative vector of the products' demand on the market, and $\bar{P}_{R}(t) \geq 0$ as a non-negative vector of the products' prices on the market. Each non-negative component $\left[V_{S}(t)\right]_{i} \geq 0$ of the vector $\bar{V}_{S}(t) \geq 0$ represents the supply on the market for particular product; each non-negative component $\left[V_{D}(t)\right]_{i} \geq 0$ of the vector $\bar{V}_{D}(t) \geq 0$ represents the demand on the market for particular product; and each non-negative component $\left[P_{R}(t)\right]_{i} \geq 0$ of the vector $\bar{P}_{R}(t) \geq 0$ represents the price on the market for particular product $(i=1, \ldots, n)$.

As earlier, when there are no disturbing economic forces, the market is in equilibrium position for each position, i.e. the product's supply and demand are equal for every individual product, and they are developing with constant rates and all the product's prices are fixed.

When the balance between the product's supply and demand is broken for any particular product, the product's market is experienced the economic forces, which act to bring the market to a new equilibrium position. 
These economic forces are described by the following ordinary differential equations regarding to each individual product of vectors of the product's supply $\bar{V}_{S}(t)$, demand $\bar{V}_{D}(t)$, and price $\bar{P}_{R}(t)$.

$$
\begin{aligned}
& \frac{d\left[P_{R}(t)\right]_{i}}{d t}=-\left[\lambda_{P}\right]_{i i}\left(\left[V_{S}(t)\right]_{i}-\left[V_{D}(t)\right]_{i}\right) \\
& \frac{d^{2}\left[V_{S}(t)\right]_{i}}{d t^{2}}=\left[\lambda_{S}\right]_{i i} \frac{d\left[P_{R}(t)\right]_{i}}{d t} \\
& \frac{d^{2}\left[V_{D}(t)\right]_{i}}{d t^{2}}=-\left[\lambda_{D}\right]_{i i} \frac{d^{2}\left[P_{R}(t)\right]_{i}}{d t^{2}}
\end{aligned}
$$

In Equations $(1.138-140)$ above the values $\left[\lambda_{P}\right]_{i i},\left[\lambda_{S}\right]_{i i},\left[\lambda_{D}\right]_{i i} \geq 0$ are constants.

I assume that the markets had been in equilibrium position until time $t=t_{0}$, the vectors of volumes of product's supply $\bar{V}_{S}(t)$ and demand $\bar{V}_{D}(t)$ on the markets were equal, and they both were developing with vector of constant rates $\bar{r}_{D}^{0}$.

$$
\begin{aligned}
& \bar{V}_{D}(t)=\bar{r}_{D}^{0}\left(t-t_{0}\right)+\bar{V}_{D}^{0} \\
& \bar{V}_{S}(t)=\bar{V}_{D}(t)
\end{aligned}
$$

where $\bar{V}_{D}\left(t_{0}\right)=\bar{V}_{D}^{0}$.

I present below two scenarios describing the situation with withdrawal of products' savings.

\section{A Constant-Rate Continuous Withdrawal of Product Savings from Market}

According to this scenario I assume that vector of products' savings $\bar{S}_{R}(t)$ increases since time $t=t_{0}$ according to following formula in vector form,

$$
\bar{S}_{R}(t)=\left\{\begin{array}{cc}
\overline{0}, & t<t_{0} \\
\bar{\delta}_{R}\left(t-t_{0}\right), & t \geq t_{0}
\end{array}\right.
$$


where $\bar{S}_{R}(t)=\overline{0}$ for $t<t_{0}$ and $\bar{\delta}_{R} \geq \overline{0}$.

Hence taken into account the products' withdrawal from the markets in the form of products' savings $\bar{S}_{R}(t)$ described by Equation (1.143) the vector of products' surpluses (or shortages) on the markets $\left[\bar{V}_{S}(t)-\bar{V}_{D}(t)\right]$ expressed component-wise in Equations $(1.138-140)$ has to be replaced by the vector of products' surpluses (or shortages) on markets expressed as $\bar{D}_{R} \equiv\left(\bar{V}_{S}(t)-\bar{V}_{D}(t)-\bar{S}_{R}(t)\right)$; that produces the following matrix equation for $t>t_{0}$,

$$
\frac{d^{2} \bar{D}_{R}(t)}{d t^{2}}+\Lambda_{P} \Lambda_{D} \frac{d \bar{D}_{R}(t)}{d t}+\Lambda_{P} \Lambda_{S} \bar{D}_{R}(t)=\overline{0}
$$

with the following initial conditions,

$$
\begin{aligned}
& \bar{D}_{R}\left(t_{0}\right)=\overline{0}, \\
& \frac{d \bar{D}_{R}\left(t_{0}\right)}{d t}=-\bar{\delta}_{R} .
\end{aligned}
$$

In matrix Equation (1.144) above the matrices $\Lambda_{P}, \Lambda_{S}, \Lambda_{D}$ are non-negative diagonal matrices.

Initial conditions for the vector of products' prices $\bar{P}_{R}(t)$ are $\bar{P}_{R}\left(t_{0}\right)=\bar{P}_{R}^{0}$ and $\frac{d \bar{P}_{R}\left(t_{0}\right)}{d t}=\overline{0}$.

Similar to Equation (1.144) the vector of products' prices $\bar{P}_{R}(t)$ is described by the following matrix equation for $t>t_{0}$,

$$
\frac{d^{2} \bar{P}_{R}(t)}{d t^{2}}+\Lambda_{P} \Lambda_{D} \frac{d \bar{P}_{R}(t)}{d t}+\Lambda_{P} \Lambda_{S} \bar{P}_{R}(t)+\bar{C}=\overline{0}
$$

where $\bar{C}=-\Lambda_{P}\left(\Lambda_{S} \bar{P}_{R}^{0}+\bar{\delta}_{R}\right)$ is a constant vector.

If one uses the new vector $\bar{P}_{1}(t)=\bar{P}_{R}(t)-\bar{P}_{R}^{0}-\Lambda_{S}^{-1} \bar{\delta}_{R}$, Equation (1.145) becomes,

$$
\frac{d^{2} \bar{P}_{1}(t)}{d t^{2}}+\Lambda_{P} \Lambda_{D} \frac{d \bar{P}_{1}(t)}{d t}+\Lambda_{P} \Lambda_{S} \bar{P}_{1}(t)=\overline{0}
$$

Therefore the initial conditions for vector $\bar{P}_{1}(t)$ are $\bar{P}_{1}\left(t_{0}\right)=-\Lambda_{S}^{-1} \bar{\delta}_{R}$ and $\frac{d \bar{P}_{1}\left(t_{0}\right)}{d t}=\overline{0}$. 
As was shown in the model of single product economy, each component of the vectors $\bar{D}_{R}(t)$ and $\bar{P}_{1}(t)$ approaches zero with the passage of time, $\left[\bar{D}_{R}(t)\right]_{i} \rightarrow 0$ and $\left[\bar{P}_{1}(t)\right]_{i} \rightarrow 0$ for $t \rightarrow+\infty$. Thus it takes place $D_{R}(t) \rightarrow 0$ and $P_{1}(t) \rightarrow 0$ for $t \rightarrow+\infty$.

Therefore from the change of vector it takes place for $t \rightarrow+\infty$,

$$
\bar{P}_{R}(t) \rightarrow \bar{P}_{R}^{0}+\Lambda_{S}^{-1} \bar{\delta}_{R}
$$

Since $\bar{V}_{S}\left(t_{0}\right)=\bar{V}_{D}^{0}$ and $\frac{d \bar{V}_{S}\left(t_{0}\right)}{d t}=\bar{r}_{D}^{0}$ it takes place for $t \rightarrow+\infty$ from Equations $(1.138-140)$ similar to the model of single product economy,

$$
\begin{aligned}
& \bar{V}_{S}(t) \rightarrow\left(\bar{r}_{D}^{0}+\bar{\delta}_{R}\right)\left(t-t_{0}\right)+\bar{V}_{D}^{0}-\Lambda_{D} \Lambda_{S}^{-1} \bar{\delta}_{R} \\
& \bar{V}_{D}(t) \rightarrow \bar{r}_{D}^{0}\left(t-t_{0}\right)+\bar{V}_{D}^{0}-\Lambda_{D} \Lambda_{S}^{-1} \bar{\delta}_{R} \\
& \bar{S}_{R}(t)=\bar{\delta}_{R}\left(t-t_{0}\right)
\end{aligned}
$$

I calculate now the effect of the products' savings by comparing two monetary values of vectors of products taken at the limit $t \rightarrow+\infty$ i.e. when the markets of products come to a new equilibrium. The first monetary value is scalar product $v_{R}=\bar{P}_{R}(t) \times \bar{V}_{D}(t)=\sum_{i=1}^{n}\left[P_{R}(t)\right]_{i}\left[V_{D}(t)\right]_{i}$ of the vector of products' prices $\bar{P}_{R}(t)$ and the vector of products' demand $\bar{V}_{D}(t)$ at the limit after savings. The second monetary value is scalar product $\widetilde{v}_{R}=\widetilde{P}_{R}(t) \times \widetilde{V}_{D}(t)$ of the vector of products' prices $\widetilde{P}_{R}(t)=\bar{P}_{R}^{0}$ and the vector of products' demand $\widetilde{V}_{D}(t)=\bar{r}_{D}^{0}\left(t-t_{0}\right)+\bar{V}_{D}^{0}$ at the limit if there was no withdrawal of products from the markets.

Therefore at the limit for $t \rightarrow+\infty$ the withdrawal of product from the market causes an increase of that product's price.

$$
\lim _{t \rightarrow+\infty}\left(\frac{\left[P_{R}(t)\right]_{i}}{\left[\widetilde{P}_{R}(t)\right]_{i}}\right)=1+\frac{\left[\delta_{R}\right]_{i}}{\left[\lambda_{S}\right]_{i i}\left[P_{R}^{0}\right]_{i}} \geq 1
$$


Since at the limit for $t \rightarrow+\infty$ the withdrawal of product from the market practically doesn't change the vector of products' demand, the monetary value $v_{R}(t)$ of the vector of products on the market will increase in the long run,

$$
\lim _{t \rightarrow+\infty}\left(\frac{v_{R}(t)}{\widetilde{v}_{R}(t)}\right)=\lim _{t \rightarrow+\infty}\left(\frac{\bar{P}_{R}(t) \times \bar{V}_{D}(t)}{\widetilde{P}_{R}(t) \times \widetilde{V}_{D}(t)}\right)=\frac{\lim _{t \rightarrow+\infty}\left(\sum_{i=1}^{n}\left[P_{R}(t)\right]_{i}\left[r_{D}^{0}\right]_{i}\right)}{\sum_{i=1}^{n}\left[P_{R}^{0}\right]_{i}\left[r_{D}^{0}\right]_{i}}>1
$$

as it takes place for each component, $\lim _{t \rightarrow+\infty}\left[P_{R}(t)\right]_{i} \geq\left[P_{R}^{0}\right]_{i}$ for $\left[\delta_{R}\right]_{i}>0, i=1, \ldots, n$.

That concludes the first scenario.

\section{B Constant-Accelerated Continuous Withdrawal of Product Savings from Market}

According to this scenario I assume that vector of products' savings $\bar{S}_{R}(t)$ increases since time $t=t_{0}$ according to following formula in vector form,

$$
\bar{S}_{R}(t)=\left\{\begin{array}{cc}
\overline{0}, & t<t_{0} \\
\overline{\delta_{R}}\left(t-t_{0}\right)+\frac{\overline{\varepsilon_{R}}}{2}\left(t-t_{0}\right)^{2}, & t \geq t_{0}
\end{array}\right.
$$

where $\bar{S}_{R}(t)=\overline{0}$ for $t<t_{0}, \bar{\delta}_{R} \geq \overline{0}$, and $\bar{\varepsilon}_{R} \geq \overline{0}$.

Hence taken into account the products' withdrawal from the markets in the form of products' savings $\bar{S}_{R}(t)$ described by Equation (1.143) the vector of products' surpluses (or shortages) on the markets $\left[\bar{V}_{S}(t)-\bar{V}_{D}(t)\right]$ expressed component-wise in Equations $(1.138-140)$ has to be replaced by the vector of products' surpluses (or shortages) on markets expressed as $\bar{D}_{R} \equiv\left(\bar{V}_{S}(t)-\bar{V}_{D}(t)-\bar{S}_{R}(t)\right)$; that produces the following matrix equation for $t>t_{0}$,

$$
\frac{d^{2} \bar{D}_{R}(t)}{d t^{2}}+\Lambda_{P} \Lambda_{D} \frac{d \bar{D}_{R}(t)}{d t}+\Lambda_{P} \Lambda_{S} \bar{D}_{R}(t)+\bar{\varepsilon}_{R}=\overline{0}
$$


with the following initial conditions,

$$
\begin{aligned}
& \bar{D}_{R}\left(t_{0}\right)=\overline{0}, \\
& \frac{d \bar{D}_{R}\left(t_{0}\right)}{d t}=-\bar{\delta}_{R} .
\end{aligned}
$$

In matrix Equation (1.154) above the matrices $\Lambda_{P}, \Lambda_{S}, \Lambda_{D}$ are non-negative diagonal matrices.

If one uses the new vector of products $\bar{D}_{1}(t)=\bar{D}_{R}(t)+\Lambda_{S}^{-1} \Lambda_{P}^{-1} \bar{\varepsilon}_{R}$, then Equation (1.154) becomes,

$$
\frac{d^{2} \overline{D_{1}}(t)}{d t^{2}}+\Lambda_{P} \Lambda_{D} \frac{d \overline{D_{1}}(t)}{d t}+\Lambda_{P} \Lambda_{S} \bar{D}_{1}(t)=\overline{0}
$$

with the following initial conditions,

$$
\begin{aligned}
& \bar{D}_{1}\left(t_{0}\right)=\Lambda_{S}^{-1} \Lambda_{P}^{-1} \bar{\varepsilon}_{R}, \\
& \frac{d \bar{D}_{1}\left(t_{0}\right)}{d t}=-\bar{\delta}_{R} .
\end{aligned}
$$

Initial conditions for the vector of products' prices $\bar{P}_{R}(t)$ are $\bar{P}_{R}\left(t_{0}\right)=\bar{P}_{R}^{0}$ and $\frac{d \bar{P}_{R}\left(t_{0}\right)}{d t}=\overline{0}$.

Similar to Equation (1.154) the vector of products' prices $\bar{P}_{R}(t)$ is described by the following matrix equation for $t>t_{0}$,

$$
\frac{d^{2} \bar{P}_{R}(t)}{d t^{2}}+\Lambda_{P} \Lambda_{D} \frac{d \bar{P}_{R}(t)}{d t}+\Lambda_{P} \Lambda_{S} \bar{P}_{R}(t)-\Lambda_{P}\left(\bar{\varepsilon}_{R}\left(t-t_{0}\right)+\bar{\delta}_{R}+\Lambda_{S} \bar{P}_{R}^{0}\right)=\overline{0}
$$

If one uses new vector $\bar{P}_{1}(t)=\bar{P}_{R}(t)-\bar{P}_{R}^{0}-\Lambda_{S}^{-1} \bar{\delta}_{R}-\Lambda_{S}^{-1} \bar{\varepsilon}_{R}\left(t-t_{0}\right)+\Lambda_{S}^{-1} \Lambda_{D} \Lambda_{S}^{-1} \bar{\varepsilon}_{R}$, then Equation (1.156) becomes,

$$
\frac{d^{2} \overline{P_{1}}(t)}{d t^{2}}+\Lambda_{P} \Lambda_{D} \frac{d \bar{P}_{1}(t)}{d t}+\Lambda_{P} \Lambda_{S} \bar{P}_{1}(t)=\overline{0}
$$

Initial conditions for $\bar{P}_{1}(t)$ are $\bar{P}_{1}\left(t_{0}\right)=-\Lambda_{S}^{-1} \bar{\delta}_{R}+\Lambda_{S}^{-1} \Lambda_{D} \Lambda_{S}^{-1} \bar{\varepsilon}_{R}$ and $\frac{d \bar{P}_{1}\left(t_{0}\right)}{d t}=-\Lambda_{S}^{-1} \varepsilon_{R}$. 
As was shown in the model of single product economy, each component of the vectors $\bar{D}_{R}(t)$ and $\bar{P}_{1}(t)$ approaches zero with the passage of time, $\left[\bar{D}_{R}(t)\right]_{i} \rightarrow 0$ and $\left[\bar{P}_{1}(t)\right]_{i} \rightarrow 0$ for $t \rightarrow+\infty$. Thus it takes place $D_{R}(t) \rightarrow 0$ and $P_{1}(t) \rightarrow 0$ for $t \rightarrow+\infty$.

Therefore from the change of vectors it takes place for $t \rightarrow+\infty$,

$$
\begin{aligned}
& \bar{D}_{R}(t) \rightarrow-\Lambda_{S}^{-1} \Lambda_{P}^{-1} \bar{\varepsilon}_{R} \\
& \bar{P}_{R}(t) \rightarrow \Lambda_{S}^{-1} \bar{\varepsilon}_{R}\left(t-t_{0}\right)+\bar{P}_{R}^{0}+\Lambda_{S}^{-1} \bar{\delta}_{R}-\Lambda_{S}^{-1} \Lambda_{D} \Lambda_{S}^{-1} \bar{\varepsilon}_{R}
\end{aligned}
$$

Since $\bar{V}_{S}\left(t_{0}\right)=\bar{V}_{D}^{0}$ and $\frac{d \bar{V}_{S}\left(t_{0}\right)}{d t}=\bar{r}_{D}^{0}$ it takes place for $t \rightarrow+\infty$ from Equations $(1.138-140)$ similar to the model of single product economy,

$$
\begin{aligned}
& \bar{V}_{S}(t) \rightarrow\left(\bar{r}_{D}^{0}+\bar{\delta}_{R}-\Lambda_{D} \Lambda_{S}^{-1} \bar{\varepsilon}_{R}\right)\left(t-t_{0}\right)+\frac{\bar{\varepsilon}_{R}}{2}\left(t-t_{0}\right)^{2}+\bar{V}_{D}^{0}-\Lambda_{D} \Lambda_{S}^{-1} \bar{\delta}_{R} \\
& -\Lambda_{S}^{-1} \Lambda_{P}^{-1} \bar{\varepsilon}_{R}+\Lambda_{D} \Lambda_{S}^{-1} \Lambda_{D} \Lambda_{S}^{-1} \bar{\varepsilon}_{R} \\
& \bar{V}_{D}(t) \rightarrow\left(\bar{r}_{D}^{0}-\Lambda_{D} \Lambda_{S}^{-1} \bar{\varepsilon}_{R}\right)\left(t-t_{0}\right)+\bar{V}_{D}^{0}-\Lambda_{D} \Lambda_{S}^{-1} \bar{\delta}_{R}+\Lambda_{D} \Lambda_{S}^{-1} \Lambda_{D} \Lambda_{S}^{-1} \bar{\varepsilon}_{R} \\
& \bar{S}_{R}(t)=\bar{\delta}_{R}\left(t-t_{0}\right)+\frac{\bar{\varepsilon}_{R}}{2}\left(t-t_{0}\right)^{2}
\end{aligned}
$$

As before I calculate the effect of the products' savings by comparing two monetary values of vectors of products taken at the limit $t \rightarrow+\infty$ i.e. when the markets of products come to a new equilibrium. The first monetary value is scalar product $v_{R}=\bar{P}_{R}(t) \times \bar{V}_{D}(t)$ of the vector of products' prices $\bar{P}_{R}(t)$ and the vector of products' demand $\bar{V}_{D}(t)$ at the limit after savings. The second monetary value is scalar product $\widetilde{v}_{R}=\widetilde{P}_{R}(t) \times \widetilde{V}_{D}(t)$ of the vector of products' prices $\widetilde{P}_{R}(t)=\bar{P}_{R}^{0}$ and the vector of products' demand $\widetilde{V}_{D}(t)$ at the limit if there was no withdrawal of products from the markets.

Therefore at the limit for $t \rightarrow+\infty$ the withdrawal of product from the market causes an unlimited increase of that product's price,

$$
\lim _{t \rightarrow+\infty}\left(\frac{\left[P_{R}(t)\right]_{i}}{\left[\widetilde{P}_{R}(t)\right]_{i}}\right)=\lim _{t \rightarrow+\infty}\left(\frac{\left[\varepsilon_{R}\right]_{i}}{\left[\lambda_{S}\right]_{i i}\left[P_{R}^{0}\right]_{i}}\left(t-t_{0}\right)\right)=+\infty
$$


when $\left[\varepsilon_{R}\right]_{i}>0$.

Thus at the limit for $t \rightarrow+\infty$ the monetary value $v_{R}(t)$ of the vector of products on the market will increase unlimitedly in the long run (as long as each savings withdrawal is carried out with a moderate acceleration rate),

$\lim _{t \rightarrow+\infty}\left(\frac{v_{R}(t)}{\widetilde{v}_{R}(t)}\right)=\lim _{t \rightarrow+\infty}\left(\frac{\bar{P}_{R}(t) \times \bar{V}_{D}(t)}{\widetilde{P}_{R}(t) \times \widetilde{V}_{D}(t)}\right)=\frac{\lim _{t \rightarrow+\infty}\left(\left(\sum_{i=1}^{n}\left[\lambda_{S}\right]_{i i}^{-1}\left[\varepsilon_{R}\right]_{i}\left(\left[r_{D}^{0}\right]_{i}-\left[\lambda_{D}\right]_{i i}\left[\lambda_{S}\right]_{i i}^{-1}\left[\varepsilon_{R}\right]_{i}\right)\right)\left(t-t_{0}\right)\right)}{\sum_{i=1}^{n}\left[P_{R}^{0}\right]_{i}\left[r_{D}^{0}\right]_{i}}=+\infty$

when it is fulfilled for each component, $\left[\varepsilon_{R}\right]_{i}<\left[\lambda_{S}\right]_{i i}\left[\lambda_{D}\right]_{i i}^{-1}\left[r_{D}^{0}\right]_{i}$ for $\left[\varepsilon_{R}\right]_{i}>0, i=1, \ldots, n$.

That concludes the second scenario.

\section{Applied Models of Multiple Product Economy}

I present here the continuous time mathematical models that are partially based on Input-Output model of Wassily Leontief (see [2] - [5]).

As in [4], [5] I am going to use in the models a non-negative square matrix $A$, which consists of non-negative elements $a_{i j} \geq 0$ showing the amount of product $i$ needed to produce a unit of product $j$. In continuous time production processes employed in the models I suppose that changes in input and output vectors of products occur simultaneously i.e. production time periods are equal to zero. If one wants to build a model for practical applications, she probably has to introduce a production time delay into the model.

Apart from Leontief's approach, the continuous time model described below utilizes a nonnegative square matrix $B$ additionally to matrix $A$, which consists of non-negative elements $b_{i j} \geq 0$. Matrix $B$ is depreciation matrix and its elements $b_{i j} \geq 0$ show the depreciation rate of product $i$ occurred during the production of product $j$ with the unit rate. 
I also use a mathematical fact that for inverse matrices $(I-A)^{-1}$ and $(I-B)^{-1}$ where $I$ is an identity matrix of size $n$ to exist and be non-negative it is necessary and sufficient that Frobenius eigenvalues (i.e. eigenvalue with a maximum module) of non-negative matrices $A$ and $B$ are less than one [1].

I present situation describing the effect of depreciation expenses on the market dynamics and two scenarios illustrating effect of products' supplies for external customers.

\section{A Continuous Depreciation Expenses and Market Dynamics}

Here I consider effect of depreciation expenses on market dynamics in multiple product economy. To solve the task I use the depreciation matrix $B \geq 0$ introduced above.

Then depreciation vector $\bar{V}_{D P}(t)$ is represented by formula,

$$
\bar{V}_{D P}(t)=B \bar{V}_{S}(t)
$$

where $\bar{V}_{S}(t)$ vector of products' supply.

Since at the equilibrium point the vector of products' supply $\bar{V}_{S}(t)$ is equal to the vector of products' demand $\bar{V}_{D}(t)$ and vector of depreciation expenses $\bar{V}_{D P}(t)$,

$$
\bar{V}_{S}(t)=\bar{V}_{D}(t)+\bar{V}_{D P}(t)=\bar{V}_{D}(t)+B \bar{V}_{S}(t)
$$

it takes place there,

$$
\bar{V}_{S}(t)=(I-B)^{-1} \bar{V}_{D}(t)
$$

Thus if I assume that in equilibrium position the vector of products' demand $\bar{V}_{D}(t)$ on the markets is developing with vector of constant rates $\bar{r}_{D}^{0}$,

$$
\bar{V}_{D}(t)=\bar{r}_{D}^{0}\left(t-t_{0}\right)+\bar{V}_{D}^{0}
$$

then vector of products' supply $\bar{V}_{S}(t)$ on the markets is also developing with the constant rates, 


$$
\begin{aligned}
& \frac{d \bar{V}_{S}(t)}{d t}=(I-B)^{-1} \bar{r}_{D}^{0} \\
& \bar{V}_{S}(t)=(I-B)^{-1} \bar{r}_{D}^{0}\left(t-t_{0}\right)+\bar{V}_{S}^{0}
\end{aligned}
$$

where $\bar{V}_{S}\left(t_{0}\right)=\bar{V}_{S}^{0}=(I-B)^{-1} \bar{V}_{D}^{0}$.

Similarly the vector of depreciation expenses $\bar{V}_{D P}(t)$ is developing with the constant rates,

$$
\bar{V}_{D P}(t)=B(I-B)^{-1} \bar{r}_{D}^{0}\left(t-t_{0}\right)+\bar{V}_{D P}^{0}
$$

where $\bar{V}_{D P}\left(t_{0}\right)=\bar{V}_{D P}^{0}=B(I-B)^{-1} \bar{V}_{D}^{0}$.

\section{B Constant-Rate Continuous External Supply of Products}

Here I consider a task when multiple product economy has to provide a constant-rate continuous supply of products to external customers. I also care about the effect of depreciation expenses on market dynamics. To solve the task I use two matrices introduced above - input matrix $A \geq 0$ and depreciation matrix $B \geq 0$.

According to this scenario the vector of products' supply for external needs $\bar{V}_{C}(t)$ and vector of totally produced products $\bar{V}_{T}(t)$ are related via formula,

$$
\bar{V}_{T}(t)=\bar{V}_{C}(t)+A \bar{V}_{T}(t)
$$

therefore it takes place there,

$$
\bar{V}_{T}(t)=(I-A)^{-1} \bar{V}_{C}(t)
$$

Since the total vector of products $\bar{V}_{T}(t)$ is what economy is required to produce in order to satisfy external needs, the vector of products' demand $\bar{V}_{D}(t)$ is equal to the total vector of products $\bar{V}_{T}(t)$ at the equilibrium point,

$$
\bar{V}_{D}(t)=\bar{V}_{T}(t)
$$


As before the depreciation vector $\bar{V}_{D P}(t)$ is represented by formula,

$$
\bar{V}_{D P}(t)=B \bar{V}_{S}(t)
$$

where $\bar{V}_{S}(t)$ vector of products' supply.

Since at the equilibrium point the vector of products' supply $\bar{V}_{S}(t)$ is equal to the vector of products' demand $\bar{V}_{D}(t)$ and vector of depreciation expenses $\bar{V}_{D P}(t)$,

$$
\bar{V}_{S}(t)=\bar{V}_{D}(t)+\bar{V}_{D P}(t)=\bar{V}_{D}(t)+B \bar{V}_{S}(t)
$$

it takes place there,

$$
\bar{V}_{S}(t)=(I-B)^{-1} \bar{V}_{D}(t)=(I-B)^{-1}(I-A)^{-1} \bar{V}_{C}(t)
$$

Thus if I assume that in equilibrium position the vector of products' supply for external needs $\bar{V}_{C}(t)$ is developing with vector of constant rates $\bar{r}_{C}^{0} \geq \overline{0}$,

$$
\bar{V}_{C}(t)=\bar{r}_{C}^{0}\left(t-t_{0}\right)+\bar{V}_{C}^{0}
$$

then the total vector of products' demand $\bar{V}_{D}(t)$ is also developing with the constant rates,

$$
\bar{V}_{D}(t)=(I-A)^{-1} \bar{r}_{C}^{0}\left(t-t_{0}\right)+\bar{V}_{D}^{0}
$$

where $\bar{V}_{D}\left(t_{0}\right)=\bar{V}_{D}^{0}=(I-A)^{-1} \bar{V}_{C}^{0}$.

Alike the vector of products' supply $\bar{V}_{S}(t)$ on the markets is developing with the constant rates,

$$
\bar{V}_{S}(t)=(I-B)^{-1}(I-A)^{-1} \bar{r}_{C}^{0}\left(t-t_{0}\right)+\bar{V}_{S}^{0}
$$

where $\bar{V}_{S}\left(t_{0}\right)=\bar{V}_{S}^{0}=(I-B)^{-1}(I-A)^{-1} \bar{V}_{C}^{0}$.

\section{Constant-Accelerated Continuous External Supply of Products}

At this time I consider a task when multiple product economy has to provide a constantaccelerated continuous supply of products to external customers. 
It means that it is assumed that in equilibrium position the vector of products' supply for external needs $\bar{V}_{C}(t)$ is developing with vector of constant accelerations $\bar{\varepsilon}_{C}^{0} \geq \overline{0}$,

$$
\bar{V}_{C}(t)=\frac{\bar{\varepsilon}_{C}^{0}}{2}\left(t-t_{0}\right)^{2}+\bar{r}_{C}^{0}\left(t-t_{0}\right)+\bar{V}_{C}^{0}
$$

then the total vector of products' demand $\bar{V}_{D}(t)$ is also developing with the constant accelerations,

$$
\bar{V}_{D}(t)=\frac{1}{2}(I-A)^{-1} \bar{\varepsilon}_{C}^{0}\left(t-t_{0}\right)^{2}+(I-A)^{-1} \bar{r}_{C}^{0}\left(t-t_{0}\right)+\bar{V}_{D}^{0}
$$

where $\bar{V}_{D}\left(t_{0}\right)=\bar{V}_{D}^{0}=(I-A)^{-1} \bar{V}_{C}^{0}$.

Correspondingly the vector of products' supply $\bar{V}_{S}(t)$ on the markets is developing with the constant accelerations,

$$
\bar{V}_{S}(t)=\frac{1}{2}(I-B)^{-1}(I-A)^{-1} \bar{\varepsilon}_{C}^{0}\left(t-t_{0}\right)^{2}+(I-B)^{-1}(I-A)^{-1} \bar{r}_{C}^{0}\left(t-t_{0}\right)+\bar{V}_{S}^{0}
$$

where $\bar{V}_{S}\left(t_{0}\right)=\bar{V}_{S}^{0}=(I-B)^{-1}(I-A)^{-1} \bar{V}_{C}^{0}$.

\section{Summary}

Thus I described mathematical models describing long-time effects of real savings on economic growth in economy.

First section contains research on long-term impact of real savings in single-product economy on dynamics of economic growth. There I presented models describing distinction of economic growths when savings are presented as either a one-time withdrawal of product, a constant-rate continuous withdrawal of product, a constant-accelerated continuous withdrawal of product, or an exponential continuous withdrawal of product from the market.

Modeling provided the following conceptual results. In case of a one-time (or occasional) savings economy experiences a limited economic growth in the long run with minor increase in the product's price and slight increase in the amount of product on the market. In case of a constant-rate continuous (or systematic) savings the effect is very similar - economy experiences a modest economic growth in the long 
run with minor increase in the product's price and stable amount of product on the market. And only in case of a constant-accelerated continuous (or systematic and increasing) savings economy can experience a steady economic growth in the long run. With constant-accelerated continuous savings there is an effect of continuous increase in the product's price and continuous decrease in the amount of product on the market. It produces a limit in the acceleration rate of internal savings - when it doesn't exceed the limit an economy experiences a continuous steady economic growth and when it exceeds the limit there is a continuous steady economic decline. Model showed that with an exponential continuous savings economy is exposed to an effect of continuous increase in the product's price and continuous decrease in the amount of product on the market. However the mutual combination of these outcomes always produces a continuous steady economic decline. The last fact tells one that for an economic growth internal savings should be invested directly into appropriate investment vehicles with exclusion from this process the interest-rate-bearing bank accounts. The inclusion of interest-rate-bearing bank accounts into a savings-investment chain is forcing an economy to suffer economic decline.

Second section contains a study of long-term impact of real savings in multiple-product economy on dynamics of economic growth. It has models describing distinction of economic growths when savings are presented as either a constant-rate continuous withdrawal of products or a constant-accelerated continuous withdrawal of products from the markets.

Modeling was performed to verify with the model of multiple-product economy conceptual results obtained above for a single-product economy. Results confirmed that in case of a constant-rate continuous savings economy experiences a modest economic growth in the long run with minor increases in the products' prices and stable amounts of products on the markets. Results showed that in case of a constantaccelerated continuous savings economy would experience a continuous steady economic growth in the long run only if acceleration rate of internal savings for every product doesn't exceed the appropriate limit for industry. Otherwise economy would undergo a continuous steady economic decline.

Third section presents modification of the model of multiple-product economy above to include there the effect of depreciation expenses and the products' supplies for external customers on the market dynamics. Modeling was done with the help of Input-Output model of Wassily Leontief [4], [5] where technological factors in economy were assumed to be constant. There were considered two scenarios for 
situation with the products' supplies for external customers - a constant-rate continuous supply of products and a constant-accelerated continuous supply of products to external customers. Third section is mostly technical in nature and shows what kind of adjustments can be made to theoretical mathematical model of multiple-product economy to make it more applicable to the practical needs. 


\section{References}

[1] F. R. Gantmacher, "The Theory of Matrices," vol. 1 and 2, Chelsea Publishing Co, New York, 1959.

[2] A. Krouglov, "Dynamics of Business Fluctuations in the Leontief-Type Economy," ewpmac/9807007, available at http://econwpa.wustl.edu.

[3] A. Krouglov, "Mathematical Dynamics of Economic Markets," Nova Science Publishers, New York, 2006.

[4] W. Leontief, "The Structure of the American Economy 1919-1939: An Empirical Application of Equilibrium Analysis," Oxford University Press, New York, 1951.

[5] W. Leontief et al., "Studies in the Structure of the American Economy," Oxford University Press, New York, 1953.

[6] I. G. Petrovski, "Ordinary Differential Equations," Prentice Hall, Englewoods Cliffs, NJ, 1966.

[7] N. S. Piskunov, "Differential and Integral Calculus," Groningen P. Noordhoff, 1965.

[8] L. S. Pontryagin, "Ordinary Differential Equations," Addison-Wesley, Reading, MA, 1962. 